\title{
Three-dimensional image reconstruction of skeletal tissue from computed tomography
}

\author{
Sundareswar Bangalore Jayashekar \\ West Virginia University
}

Follow this and additional works at: https://researchrepository.wvu.edu/etd

\section{Recommended Citation}

Jayashekar, Sundareswar Bangalore, "Three-dimensional image reconstruction of skeletal tissue from computed tomography" (2000). Graduate Theses, Dissertations, and Problem Reports. 1076.

https://researchrepository.wvu.edu/etd/1076

This Thesis is protected by copyright and/or related rights. It has been brought to you by the The Research Repository @ WVU with permission from the rights-holder(s). You are free to use this Thesis in any way that is permitted by the copyright and related rights legislation that applies to your use. For other uses you must obtain permission from the rights-holder(s) directly, unless additional rights are indicated by a Creative Commons license in the record and/ or on the work itself. This Thesis has been accepted for inclusion in WVU Graduate Theses, Dissertations, and Problem Reports collection by an authorized administrator of The Research Repository @ WVU. For more information, please contact researchrepository@mail.wvu.edu. 


\title{
Three Dimensional Image Reconstruction of Skeletal Tissue from Computed Tomography
}

\author{
Sundareswar Jayashekar \\ Masters Thesis \\ submitted to the \\ College of Engineering and Mineral Resources \\ at West Virginia University \\ in partial fulfillment of the requirements for the degree of \\ Masters of Science \\ Computer Science \\ Bojan Cukic, Ph.D., Chair. \\ Timothy Norman, Ph.D. \\ Mark Jerabek, Ph.D.
}

Computer Science \& Electrical Engineering Department

West Virginia University

Morgantown, WV 26506

2000

Keywords: Edge Detection, Image Processing, Medical Imaging, Image Segmentation.

Copyright 2000 Sundareswar Jayashekar 


\title{
Abstract \\ Three Dimensional Image Reconstruction of Skeletal Tissue from Computed Tomography
}

\author{
Sundareswar Jayashekar
}

Edge Detection Techniques play a very important role in medical imaging. Pre-operative diagnosis of diseases such as cancer, tumors, bone fractures etc. depend on edge detection techniques to identify the malignant growths. In orthopedics, edge detection techniques are used in the reconstruction of the skeletal system from modalities such as $\mathrm{MRI}$ (magnetic resonance imaging) and CT(computed tomography).

In this thesis, we develop a simple process for the three dimensional image reconstruction of the human knee, starting from computed tomography scans. Designing a digital image processing technique which successfully separates the bone from the surrounding flesh and muscle tissue is the main focus of the thesis. We use edge detection procedures to extract the edge of the bone. After the bone edge is determined, we proceed to extract the coordinates of the bone boundary. These coordinates are plotted in 3 dimensional space providing the 3D model of the human skeletal leg.

The 3D reconstruction method developed in this thesis is applicable to all skeletal tissue but is illustrated using the CT images of the human leg. 


\section{Acknowledgements}

I would like to express my sincere thanks to Dr.Bojan Cukic, my thesis advisor, for his guidance and invaluable suggestions that he provided during the entire course of my thesis work. He gave me the freedom to work and explore things. My sincere thanks to Dr. Timothy Norman, my graduate committee member and research advisor at the Health Sciences for his support and guidance.

I would like to thank Dr. Mark Jerabek, my graduate committee member for his assistance and guidance during the course of the Independent Study during the Summer which was of great help for my thesis work. I also would like to thank Dr. William Simons for his suggestions and guidance during the course of my research. He was of great help to me and it was a pleasure working with him.

I would also like to thank Dr. David Blaha, Chairman of Orthopedics Department, West Virginia University School of Medicine, for his inputs and suggestions on the research work.

Finally, I would like to thank my friends and colleagues at the MSRC (Musculo Skeletal Research Center), Ganesh Thyagarajan and Shalini Rangarajan who have been a constant source of encouragement and support. 


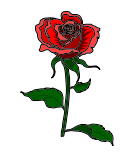

I dedicate this thesis to my parents... 


\section{Table of Contents}

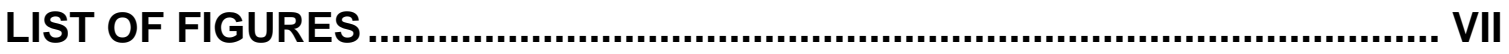

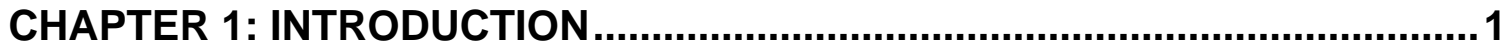

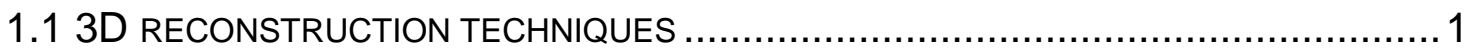

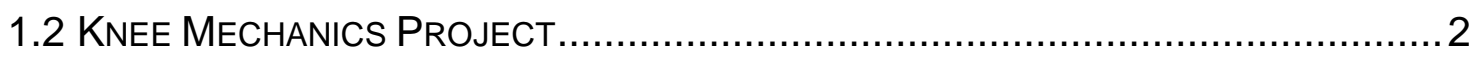

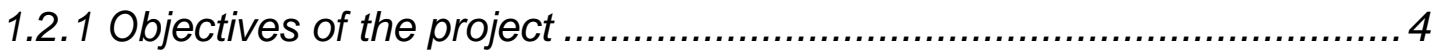

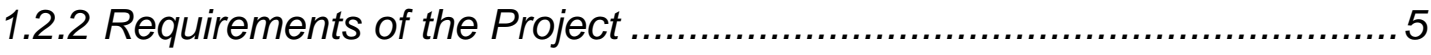

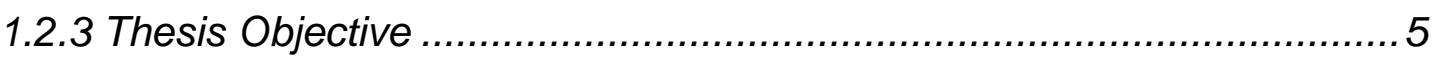

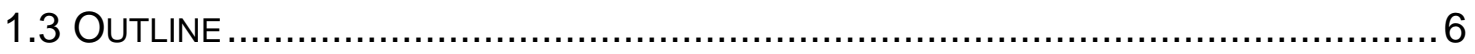

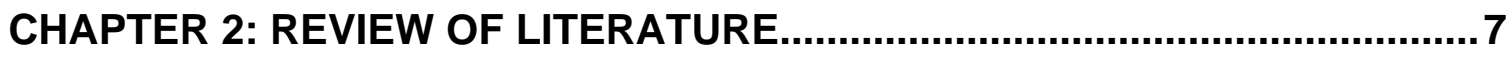

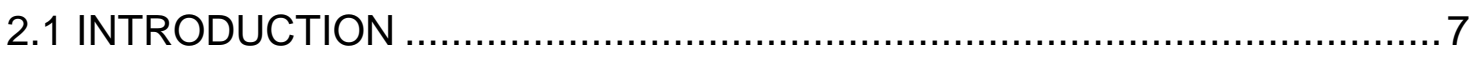

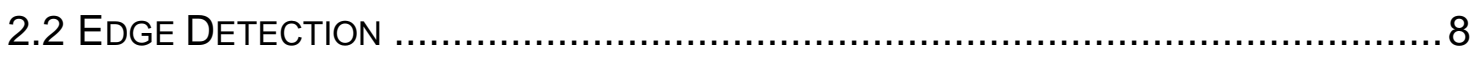

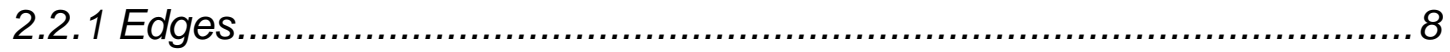

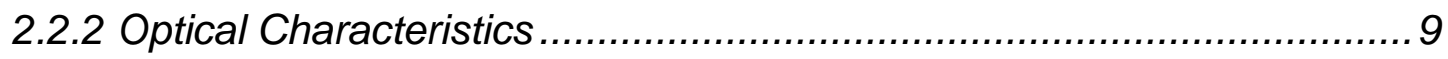

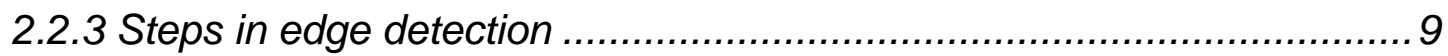

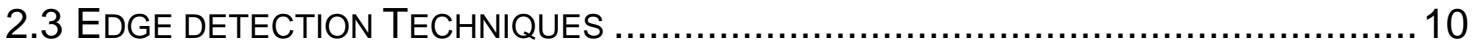

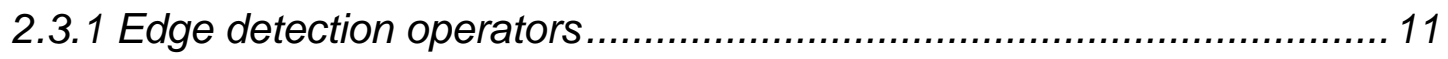

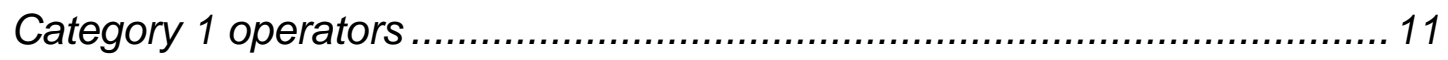

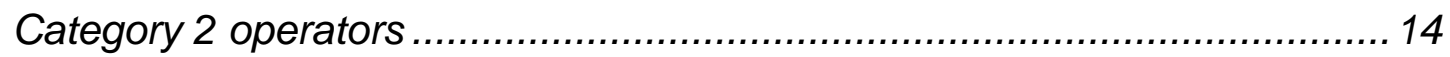

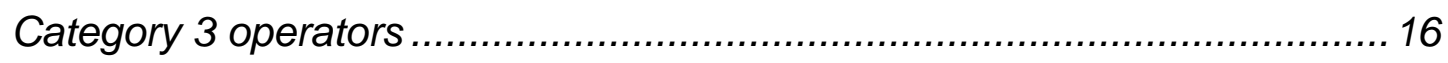

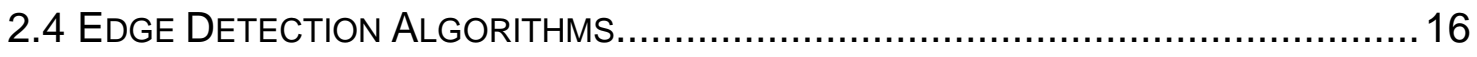

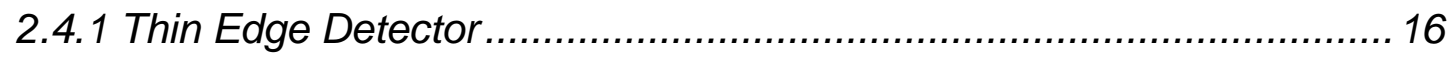

2.4.2 A Computational Approach to Edge Detection.................................... 19

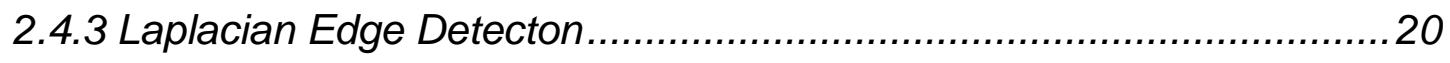

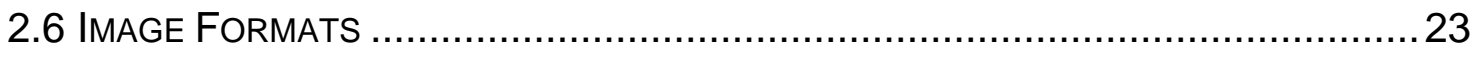

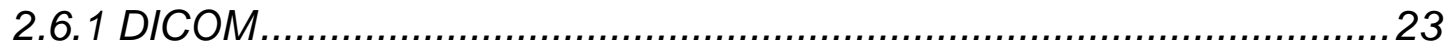

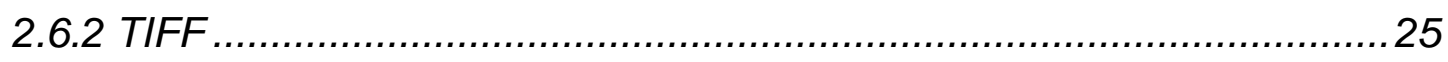

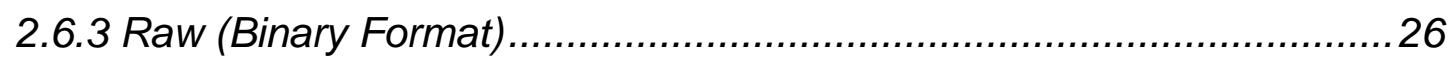

CHAPTER 3: 3D RECONSTRUCTION FROM CT...........................................28 


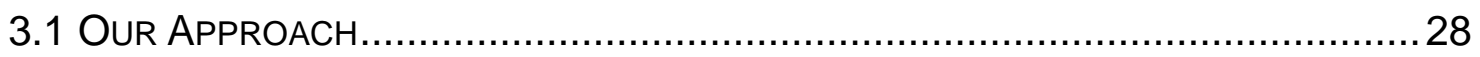

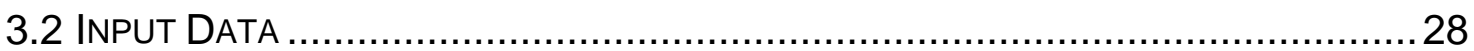

3.3 THREE DIMENSIONAL RECONSTRUCTION FROM CT SCANS …......................... 31

3.3.1 Conversion of CT's from Dicom to Tiff: ........................................... 31

3.3.2 Processing the Tiff Images to obtain the Raw (binary) Images............ 32

3.3.3 Extraction of bone-boundary coordinates from Binary Images .............33

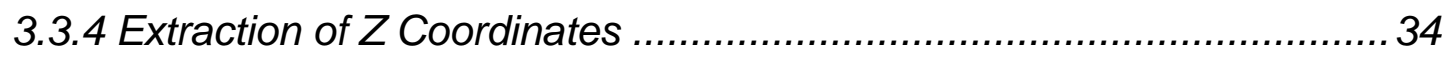

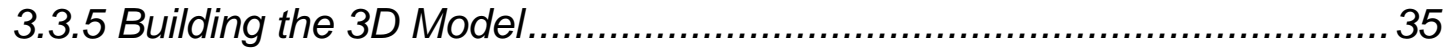

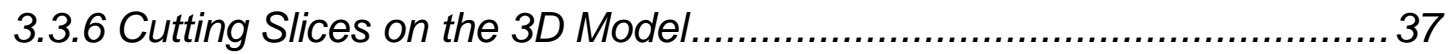

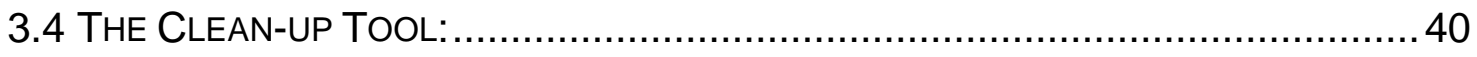

3.5 OPERATIONS ALLOWED WITH THE RECONSTRUCTION TOOL............................41

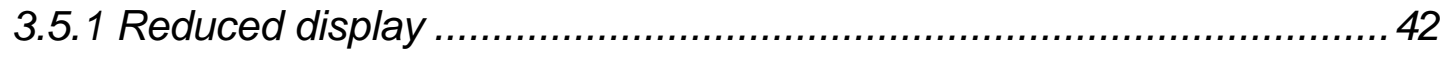

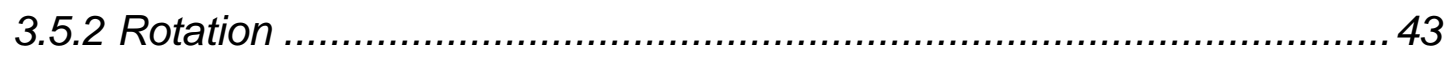

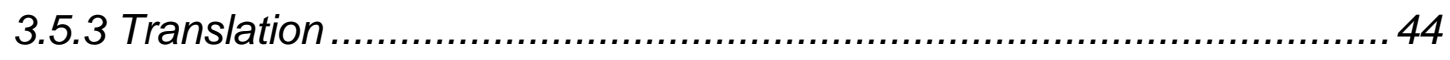

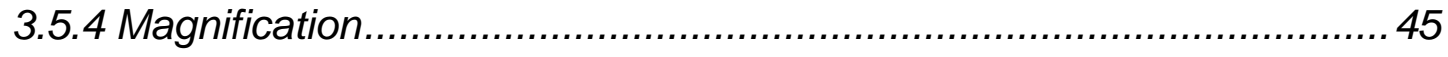

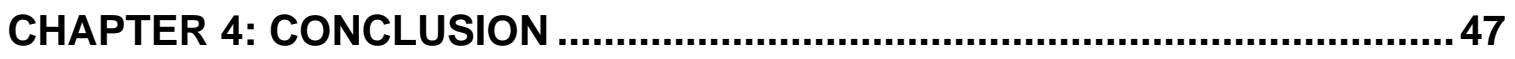

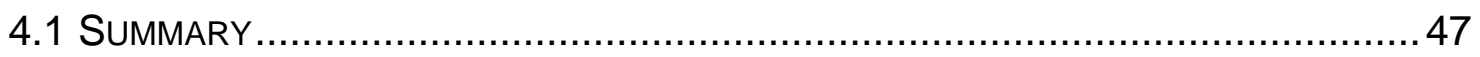

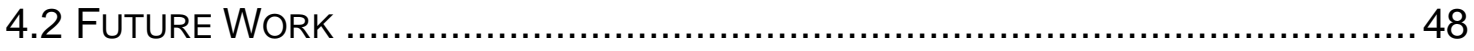

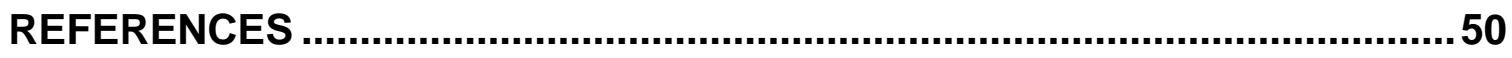

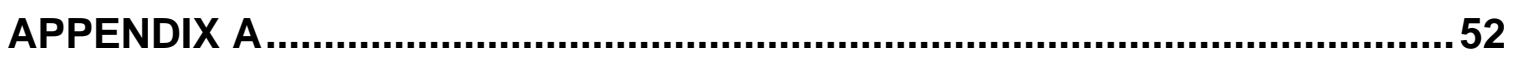

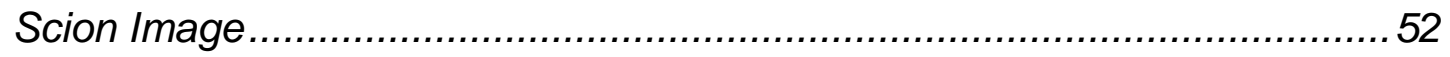

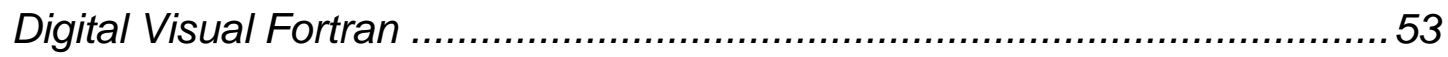

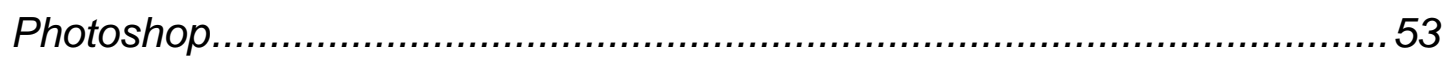

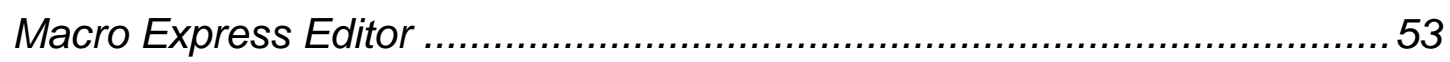

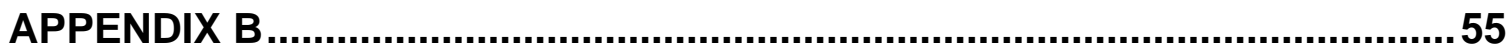

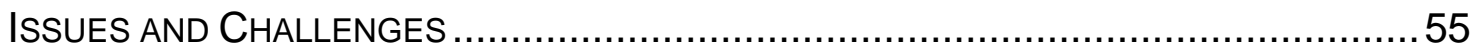

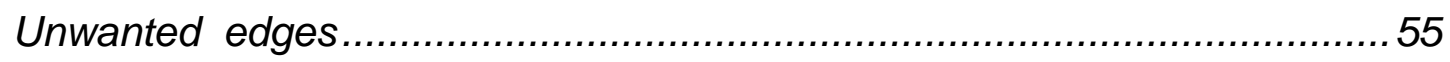

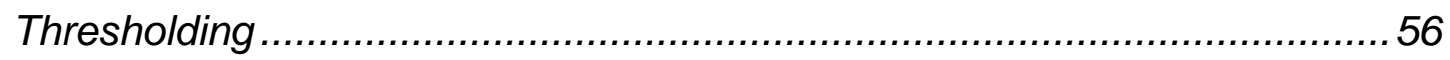

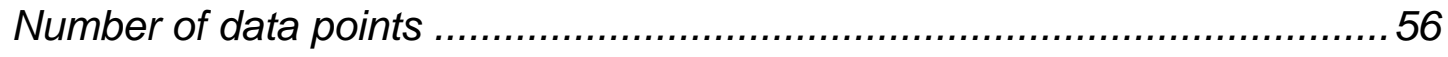




\section{List of Figures}

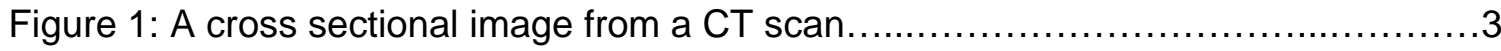

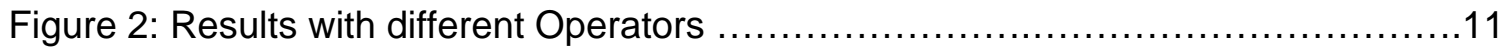

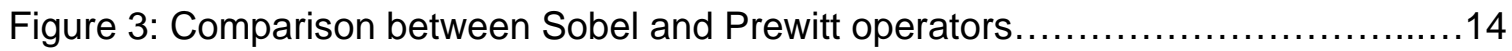

Figure 4: Gradient functions.(a)Ideal Edge, (b)Real Edge, (c)Gradient of 1 (b)...........18

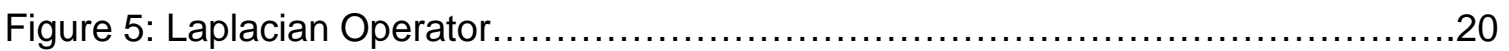

Figure 6: Gradient Operators applied to the Signal..................................

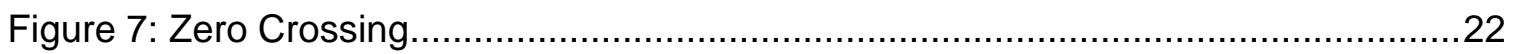

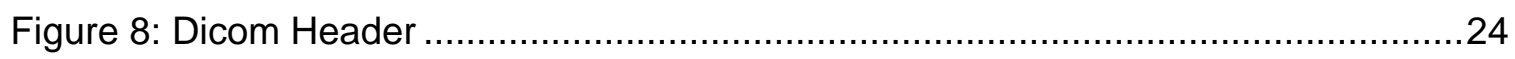

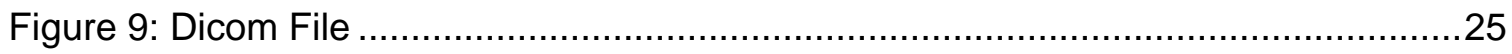

Figure 10: Sample of CT scans(a) femoral region (b) knee region (c) foot region........29

Figure 11: 3D Reconstruction from CT ............................................................... 31

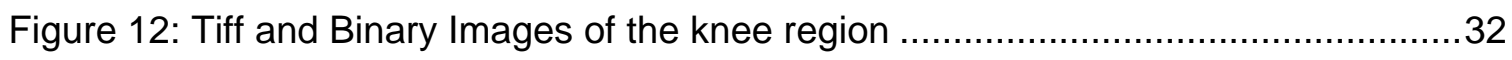

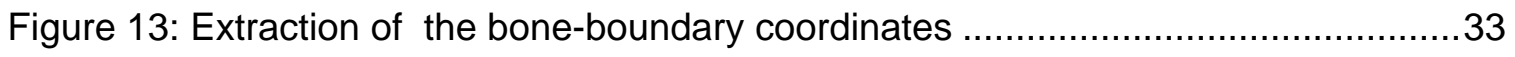

Figure 14 : A part of the Dicom Header with the $Z$ value ...........................................35

Figure 15 : Cross sectional image W.R. the $X, Y$ coordinate system ............................36

Figure 16: A 3D model showing W.R. $X, Y, Z$ coordinate system .................................36

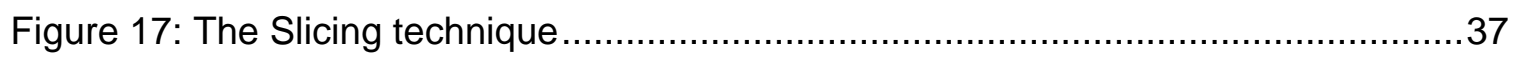

Figure 18: Cross sectional view at the knee region.................................................... 38

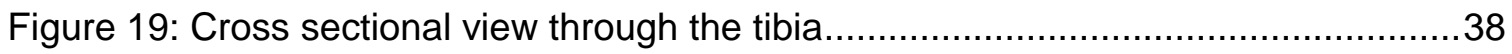

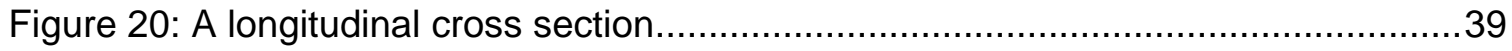

Figure 21: The box which eliminates the all the points inside it. ...................................40

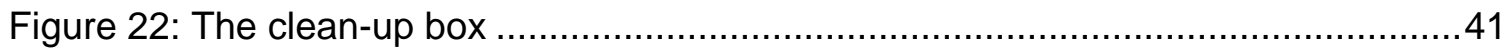

Figure 23: The model with all data points displayed.................................. 42

Figure 24: The model with one-tenth of the total number of data points.................42

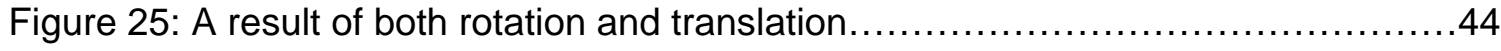

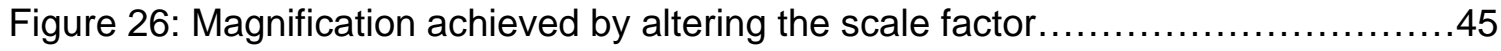

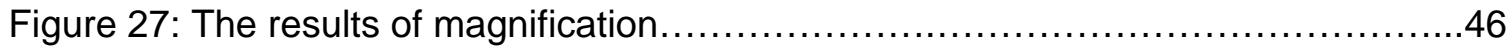

Figure 28: Macro Scripting Editor ................................................... 54 


\section{Chapter 1: Introduction}

Medical Image analysis is seen as enjoying reasonable success where quantitative issues are involved but only limited success where interpretive or diagnostic issues are involved. Systems may, for example, be able to determine the number, size and distribution of retinal microaneurysms at an acceptable accuracy, but intelligently mapping this information to a specific disease is much

more challenging. This desire for intelligent vision systems is seen also in industry. Image Analysis is a way of achieving the desired 'Intelligent Vision Systems'. Therefore Image Processing in the field of medicine is vital.

\section{$1.13 \mathrm{D}$ reconstruction techniques}

The 3D reconstruction techniques involve the building of 3D models from a two dimensional inputs such as X-rays, CT Scans, MRI Scans etc. The study done on these 3D models helps doctors in preoperative procedures and also in the better understanding of the patient's disease. This, in turn, helps in planning more appropriate and effective treatments.

There are numerous fields where the edge detection techniques and reconstruction techniques are being used. Applications of edge detection and 3D modeling techniques include:

- Identifying tumors in different parts of the body.

- Particle analysis, especially in the case of Nephrology (kidney stones, blocked canals, etc.).

- Study of brain hemorrhages. 
- Edge detection has been successfully implemented in cataract operation by ophthalmologists.

- Detection of the bone (or cartilage) from CT's and MRl's for pre-operative procedures.

The three main goals of Medical image processing applied to 3D reconstruction are:

1) To advance the state of knowledge in the mathematical theory and computer algorithms for tomographic image reconstruction and for the threedimensional(3-D) visualization and analysis of multidimensional, multiparametric, multimodality image data.

2) To develop transportable software systems for tomographic reconstruction and for 3-D visualization and analysis of medical image data.

3) To develop new medical applications for the methods of 3-D visualization and analysis for improved diagnosis, treatment, understanding and education of abnormalities in internal structures and in their function.

\subsection{Knee Mechanics Project}

It has been a constant endeavor of knee surgeons to study the motion of the knee so that better prostheses are designed and the implantation of these prostheses are done in more effective ways.

Treatment of the knee abnormalities is based on the function of the kneejoint. So the goal of this project is to define in terms of the kinematics how the human knee joint functions. The functioning of the human knee joint is defined by a set of axes know as the 'Screw Axes'. These axes define both the rotatory 
motion as well as the translatory motion of the human knee. In determining these axes, only the initial and the final positions of the knee is important.

By kinematics we mean, how one bone moves relative to another. First we try and define how the knee moves in the 'normal' state and then we study its movement in the certain pathological states. The next step is to mimic an operative therapy and then see how it moves after the operation. These different sets of analysis help in determining which treatment works better than the rest.

Since the input data is in the form of CT Scans, we will explain in the following paragraph what a CT image is.

Computed Tomography $(\mathrm{CT})$ is based on the X-ray principal. As x-rays pass through the body they are absorbed or attenuated (weakened) at differing levels creating a matrix or profile of $\mathrm{x}$-ray beams of different strength. This $\mathrm{x}$-ray

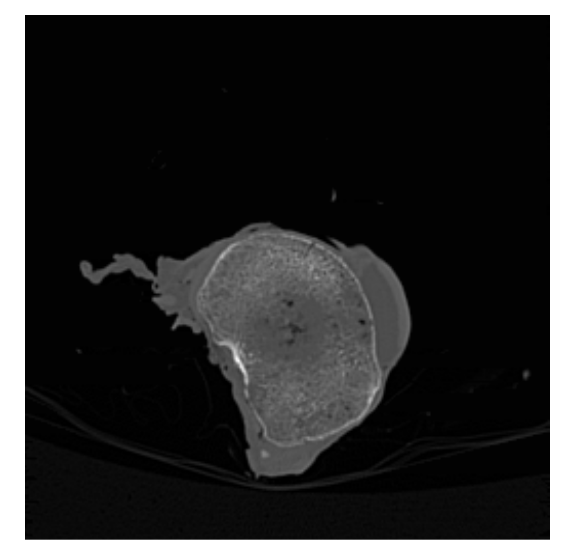

Figure 1: A cross sectional image from a CT scan

profile is registered on film, thus creating an image. In the case of CT, the film is replaced by a semi-circular detector which measures the x-ray profile. CTs comprises of the actual images, in gray scale(unlike images generated by 
attenuation of $x$-rays) of the cut cross-sections of the scanned region. Figure 1 shows a cross sectional image from a CT Scan showing the bone and the surrounding muscle tissue.

\subsubsection{Objectives of the project}

Data relating to the motion of the human knee has been collected over the years through the 'Knee Kinematics Research'. These data sets have been collected from experiments on cadaver bones. Analysis of the data would prove that there is a well defined axis of motion that the knee follows. The results of the above analysis could be shown 'visually' with the help of the 3D model. If the knee-motion-axis is identified and well understood, then it would change the way surgeries are done in the implantation of artificial knees. The best way to analyze the two dimensional data would be to covert it to $3 \mathrm{D}$, so that the results are realistic (a picture is better than a thousand words). What we need, is a way to display the results in an understandable way.

It is proposed that there exists an axis that passes through the 'Neck' of the femur and the 'Condular Groove' in the knee joint [4] and the 'Talus Bone' on the foot. It is the same axis which determines the knee motion. To prove that both of the above axes are one and the same, we would have to do the following:

- Display the data of the human knee in the form of a 3D model.

- Allow the knee motion in the 3D model.

- Recalculate the new 'Screw Axis'.

- Check if the new screw axis passes through the proposed points on the knee anatomy. 


\subsubsection{Requirements of the Project}

The project requires a 3D model of the human skeletal leg. This model is to be developed from Computed Tomography Scans. The reconstructed model should be such that the $X Y Z$ coordinates of various points on the model must be available. The XYZ coordinates help in the calculation of the 'Functional Axis' or the 'Screw Axis' of the human knee. The axes are calculated by observing definite 'marker beads' on the human bone, when the bone is subjected to motion. The positions of the 'marker beads' are noted by a 3D digitizer. The calculation of the 'Screw Axis' is beyond the scope of this thesis work. For further reading on Screw Axis please read [5].

The 3D model would be used to study the pattern that is traced by the 'Screw Axis' when the human knee is subjected to different kinds of motions. A study of the pattern of the axis would result in a better understanding of the knee motion. Slicing of the 3D model should be made possible so as to be able to view a generic cross-section of the 3D model. This would help in seeing the articulating surfaces of the bones in contact.

\subsubsection{Thesis Objective}

The objective of the thesis is to develop a tool by which 3D reconstruction of the human skeletal leg can be built from computed tomography scans. The $X$, $Y$ and $Z$ coordinates of all the points on the model should be available for further analysis, such as calculation of the 'screw axis'.

In developing the reconstruction process, stress is laid on the simplicity of the method and time efficiency. According to the process described in this thesis, 
it takes just 2 days, about 10 man-hours to process each set of knee data. It is a vast improvement over the previous method which took about a month to do a single specimen. Previously, the edge of the bone was detected manually by hand tracing. Coordinates of the bone boundary were found by manual digitization. A person had to trace the edge of the bone and note the coordinates of the bone. This process was tedious and time consuming.

The reconstruction process developed in this thesis is much faster and requires less human intervention.

\subsection{Outline}

The rest of the thesis is organized as follows.

Chapter 2 gives an overview of the existing methods for edge detection. There is a brief description of the operators used in the edge detection techniques. Common edge detection algorithms are discussed in this chapter. An introduction to the various image formats is also given.

Chapter 3 presents our methodology for the 3D reconstruction of the human skeletal leg from CT scans. A comparison of our approach and the existing methods is done highlighting the advantages and disadvantages. This chapter also talks about the slicing techniques used to get a cross sectional view of the model when sliced in a particular plane.

Chapter 4 summarizes the work that has been done in the context of this research project, and outlines avenues for future research work. It also throws light on some of the issues and challenges that were dealt with in this project (Appendix B). 


\section{Chapter 2: Review of Literature}

Digital image processing is concerned with the manipulation and analysis of images discretized from continuous signals [11]. Algorithms to manipulate these images mathematically involve integrals, however, the necessity of sampling these images for computer use simplifies these formulae in terms of replacing integration operations by summation operations.

\subsection{INTRODUCTION}

Edge and feature detection are two of the most common operations in image processing. Digital edge detection is a fundamental process in the computer vision area, which involves the process of determining the edges in an image.

"Edges" are points or regions in an image where there is a sharp change in the intensity function [10]. In other words edges are pixels where this function changes abruptly. The change in the intensity function usually occurs at the boundaries of images across which there is a sharp image contrast.

An image function $f(x, y)$, depends on two variable-coordinates $x$ and $y$ in the image plane. An image function describes in terms of the coordinates, $x$ and

$y$, the behavior of the image attributes like brightness, color intensity etc. A change of the image function can be described by a 2D gradient that points in the direction of the largest growth (or spatial change) of the image function.

An edge can also be defined as a property attached to an individual pixel and is calculated from the image function behavior in the neighborhood of a pixel. 


\subsection{Edge Detection}

Before we start with the edge detection techniques we present some basic concepts in edge detection.

\subsubsection{Edges}

In modern image processing systems, one of the most important preprocessing steps is to partition the image into its constituent portions. This process is normally known as image segmentation and is generally achieved by one of two methods:

- Region based methods, and

- Boundary based methods.

Region based methods normally operate by detection of similarities in gray pixel values (or any other measure of similarity). Boundary based methods work by detecting discontinuities in the gray level values of the image pixels. In either case, the ultimate aim should be the partitioning of an image into parts of similar characteristics which, hopefully, correspond to actual physical objects or characteristics of objects in the actual image scene.

As this project deals with edge detection, only boundary based methods will be considered. Most of the edge detectors are based on finding pixels with an abrupt intensity change between them and their neighbors. That is what characterizes an edge. 


\subsubsection{Optical Characteristics}

In computer vision, edges are perceived between adjacent regions of differing optical characteristics. There are three kinds of optical characteristics [9]:

1. Contrast is the local change in brightness and is defined as the difference between the average brightness of two regions.

2. Color is a function of the wavelength of the light waves arriving at the sensor.

3. Texture is commonly defined as complex visual patterns, which in turn are composed of entities or sub-patterns, with characteristic brightness', colors and sizes. At times, edges can be between regions which are entirely identical as far as the first two features are concerned but are different in terms of the texture. These are known as textural edges [9].

In this project we will be concerned with grayscale images only, hence color as a parameter will not be considered.

\subsubsection{Steps in edge detection}

An edge detection algorithm typically consists of three main processing steps:

1. Filtering involves preparing the image for edge detection. Most gradient computations based upon two or three points are highly susceptible to noise or even other flaws in the digital image representation process, such as quantization loss (loss of information that occurs due to digitization) [7]. Filtering is commonly used to improve the performance of an edge detector with respect to noise. The effect of the filter is to smooth out the locality being filtered, hence removing noise. The problem with this is that there is a trade- 
off between edge strength and noise reduction. The stronger the smoothing effect applied to the image, the greater the resulting loss in edge strength.

2. Enhancement is the process of enhancing the areas of discontinuity within the image. This is normally achieved using a differential operator. Since there is no direct way to differentiate discrete pixel values in a digital grid, this step is usually done by calculating the pixel change in the locality of the point being measured. The way that this is done varies among different algorithms [7].

3. Detection is the process of detecting the presence of edges. In simple algorithms, this is achieved by simply thresholding the enhanced image. Any edge points with magnitudes greater than the specified threshold are taken as an edge element.

As we shall see, there exist a large number of different edge detection algorithms, which implement the three steps described above, though in varying ways. The variation in the implementation of the steps above leads to similarly varying results from these algorithms. As it turns out, no one edge detection algorithm seems to give optimum results with all the different profiles of edges that might be encountered. Hence comes the need for an algorithm that takes results from all the different algorithms, fuses them and exploits the strong points of all the individual algorithms.

\subsection{Edge detection Techniques}

The edge detection techniques discussed later in this chapter make use of standard operators such as Sobel, Prewitt, Laplacian, etc., for edge finding. First 
we look at the mathematical details of each of these operators and then venture into the edge finding techniques.

\subsubsection{Edge detection operators}

Edge detection operators can be divided into three categories $[11,16]$. They are described below.

\section{Category 1 operators}

Category 1 operators approximate the spatial-derivatives of the image function using differences. Some of them are rotationally invariant (e.g. Laplacian) and thus are computed from one convolution mask only. Others, that approximate first derivatives, use several masks. The orientation is estimated on the basis of the best matching of several simple patterns. Operators approximating the first derivative of an image function are sometimes called compass operators because of their ability to determine the gradient direction.

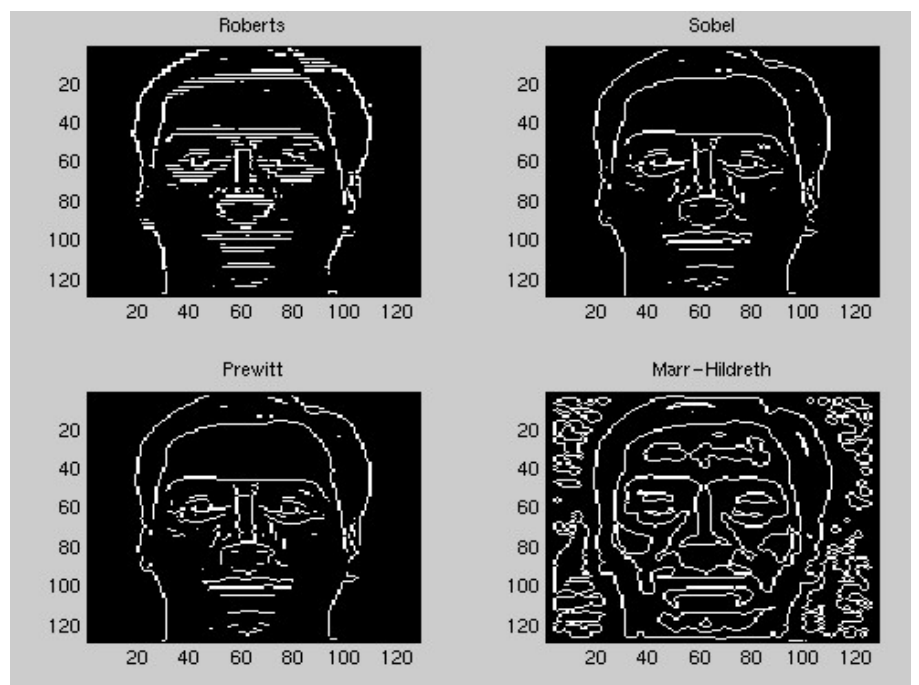

Figure 2: Results with different Operators 
Roberts operator:

The Roberts operator is one of the oldest operators. It is easy to compute as it uses only a $2 \mathrm{X} 2$ neighborhood of the current pixel. Its convolution masks are

$$
\left[\begin{array}{cc}
1 & 0 \\
0 & -1
\end{array}\right] \text { and } \quad\left[\begin{array}{cc}
0 & 1 \\
-1 & 0
\end{array}\right]
$$

so the magnitude of the edge is computed as

$|g(i, j)-g(i+1, j+1)|+|g(i, j+1)-g(i+1, j)|$, where $g$ is the image function.

The primary disadvantage of Roberts's operator is its high sensitivity to noise, because very few pixels are used to approximate the gradient [13]. The noise sensitivity of Robert's operator can be seen in Figure 2. The regions around the eye and the mouth of the picture are blurred due to the noise content in the original image.

Laplacian operator:

The Laplacian operator $\nabla^{2}$ is a popular operator approximating the second derivative, which gives the gradient magnitude only. The Laplacian equation is approximated in digital images by a convolution sum. A $3 \times 3$ mask $h$ is often used for a 4-neighborhood and a 8-neighborhood and it is defined as,

$$
\mathrm{h}=\left[\begin{array}{ccc}
0 & 1 & 0 \\
1 & -4 & 1 \\
0 & 1 & 0
\end{array}\right] \quad \text { and } \mathrm{h}=\left[\begin{array}{ccc}
1 & 1 & 1 \\
1 & -8 & 1 \\
1 & 1 & 1
\end{array}\right]
$$

respectively.

The Laplacian operator has the disadvantage that it responds doubly to some edges in the image. 
Prewitt operator:

The Prewitt operator approximates the first derivative. The gradient is estimated in eight (for a 3X3-convolution mask) possible directions and the convolution result of greatest magnitude indicates the gradient direction. Larger masks are possible.

Following is a $3 \times 3$ mask for Prewitt operator,

$$
h_{1}=\left[\begin{array}{ccc}
1 & 1 & 1 \\
0 & 0 & 0 \\
-1 & -1 & -1
\end{array}\right] \quad h_{2}=\left[\begin{array}{ccc}
0 & 1 & 1 \\
-1 & 0 & 1 \\
-1 & -1 & 0
\end{array}\right] \quad h_{3}=\left[\begin{array}{ccc}
-1 & 0 & 1 \\
-1 & 0 & 1 \\
-1 & 0 & 1
\end{array}\right]
$$

Sobel operator:

The Sobel operator is often used as a simple edge detector of horizontality and verticality of edges in which case only mask $h_{1}$ and $h_{3}$ are used. If the $h_{1}$ response is $y$ and the $h_{3}$ response $x$, we can then derive the edge strength magnitude as $\left(x^{2}+y^{2}\right)^{1 / 2}$ or $|x|+|y|$ and the direction $\operatorname{as}^{-1}(y / x)$.

Following is a $3 \times 3$ mask for Sobel operator,

$$
h_{1}=\left[\begin{array}{ccc}
1 & 2 & 1 \\
0 & 0 & 0 \\
-1 & -1 & -1
\end{array}\right] \quad h_{2}=\left[\begin{array}{ccc}
0 & 1 & 2 \\
-1 & 0 & 1 \\
-2 & -1 & 0
\end{array}\right] \quad h_{3}=\left[\begin{array}{ccc}
-1 & 0 & 1 \\
-2 & 0 & 2 \\
-1 & 0 & 1
\end{array}\right]
$$


The results with Sobel and Prewitt operators are seen in Figure 3.

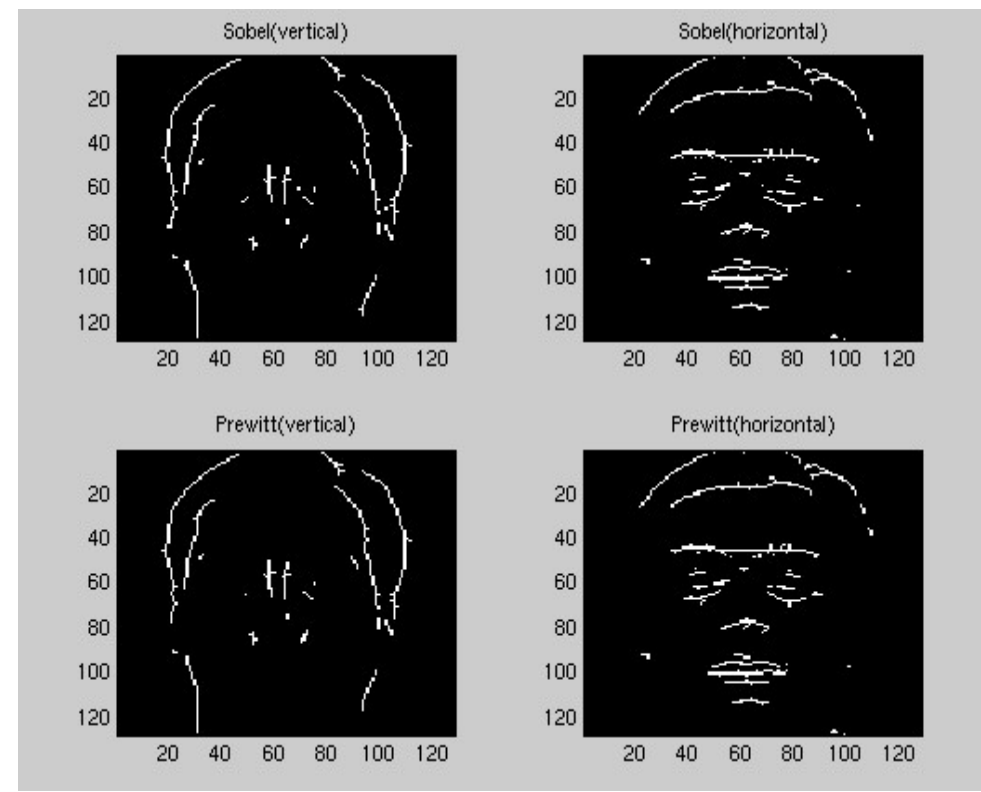

Figure 3: A Comparison between Sobel and Prewitt operators

Category 2 operators

Category 2 operators are based on the zero crossings of the second derivative of the image function (e.g. Marr-Hilderth [6] or Canny edge detector [12]). These are based on the fact that the first derivative of the image function should have an extremum at the position corresponding to the edge in the image, and so the second derivative should be zero at the same position. It is much easier and more precise to find zero crossing positions than an extremum.

Marr-Hilderth edge detector:

Proposed by Marr and Hilderth in their paper[6] in 1980, this is also called the Laplacian or the Gaussian detector. A 2D Gaussian smoothing operator $G(x, y)$ is first applied on the image to remove the noise. It is given by 
$G(x, y)=e^{-x 2+y 2 / 2 \sigma 2}$, where $x$ and $y$ are the image coordinates and $\sigma$ is the standard deviation of the associated probability distribution. The standard deviation is the only parameter of the Gaussian filter - it is proportional to the size of the neighborhood on which the filter operates. Pixels more distant from the center of the operator will have smaller influence and pixels further away than $3 \sigma$ have negligible significance. After the application of the 2D filter, Laplacian operator is used to obtain the second derivative. Finally, the convolution mask of a zero crossing detector is given by $h(x, y)=c\left(x^{2}+y^{2} / \sigma^{2}-1\right) e^{-x 2+y 2 / 2 \sigma 2}$.

Canny Edge Detector:

Canny introduces 3 criteria for edge detection [12]:

- good detection,

- good localization,

- only one response to a single edge.

The Canny operator works in a multi-stage process. First of all the image is smoothed by Gaussian convolution. Then a simple 2D first derivative operator (somewhat like the Roberts Cross) is applied to the smoothed image to highlight regions of the image with high first spatial derivatives. Edges give rise to ridges in the gradient magnitude image. The algorithm then tracks along the top of these ridges and sets to zero all pixels that are not actually on the ridge top so as to give a thin line in the output, a process known as "non-maximal suppression". The tracking process exhibits "hysteresis" controlled by two thresholds: $T 1$ and $T 2$, with $T 1>T 2$. Tracking can only begin at a point on a ridge higher than $T 1$. Tracking then continues in both directions out from that point until the height of 
the ridge falls below $T 2$. This hysteresis helps to ensure that noisy edges are not broken up into multiple edge fragments. More details about the Canny edge detector can be found in Canny's paper [12].

\section{Category 3 operators}

Category 3 operators attempt to match an image function to a parametric model of edges (e.g. snakes [1], parametrically deformable models etc). These categories of edge detectors use the higher-level image knowledge to identify regions based on a parametric template, which is deformed according to the image parameters. One example is that of a converging contour or snakes [1], which drive the contour to its minimum energy. These methods can be efficient where there exists higher-level image knowledge and pattern matches can be used. Usually these are faster than the low-level techniques.

\subsection{Edge Detection Algorithms}

The next few sections give an overview of a few edge detection algorithms that have proved useful in our research work.

\subsubsection{Thin Edge Detector}

Most edge detectors are based on detecting points in the image with a high gradient value. However, in general, they omit the idea that the edge must be a connected line of pixels. Thin Edge Detector [2] technique proposes a new edge detector, that detects entire pieces of 1-pixel thick lines. This algorithm is more robust concerning the choice of the threshold value, because it works with the average gradient value of each piece of line. 
This algorithm [2] starts with pixels with high gradient value, and follows neighbor pixels connecting new points. The thin edge detector method also solves the problem of picking up too many 'unnecessary' edges, taking advantage of the obtained information in the piece of line already detected.

The thin edge detector [2] algorithm works on gradient images which can be computed using either Roberts or Sobel masks. A minimum of a 3X3 mask size is required. Since this algorithm picks up the line (edge) by moving along the edge at 'pixel level', the image should not have much noise, and it must be as continuous as possible. Due to this, before computing gradient this algorithm uses two previous filters. The two previous filters needed are, a contrast filter to enhance the edges, and a morphological filter to remove noise but without blurring the edges of the image.

Contrast Filter : The nature of the algorithm [2] prefers the difference between the intensity values on both sides of the edge to be as high as possible. This image enhancement technique uses an optimum quantizer, according to an energy criterion, to separate the histogram of an image into subintervals, whose sizes are inversely proportional to the number of pixels affected. The result is that, an image is obtained with the most frequent gray levels are expanded whereas those gray levels with fewer number of pixels are compressed.

Morphological filter: The traditional way of removing high frequency noise is in convolving with a gaussian. This process [2] removes high frequencies in an image, just where the noise appears. However, it is precisely in these areas that 
the edges appear too. This results in a blurred image. To avoid the blurring a technique proposed by Alvarez [3] is used.

This model, based on a nonlinear diffusion, produces a selective smoothing where the edges are relatively enhanced and preserved as much as possible.

The Edge Detector: Using the gradient function $g(x)=|\nabla f(x)|$, the edge can be detected by searching the maximum gradient value.

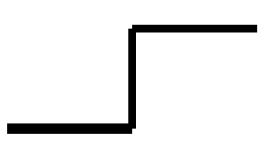

(a)

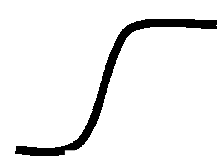

(b)

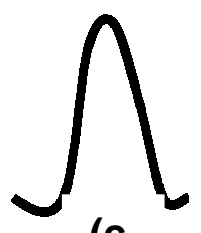

(c

Figure 4: Gradient functions - (a)Ideal Edge, (b)Real Edge, (c)Gradient of 1(b)

However, detecting points with high gradient value is not enough to obtain the entire edge line. This algorithm works similar to other contour-following algorithms. It starts from a point $P_{o}\left(x_{0}, y_{0}\right)$, with a very high gradient value. The direction of the gradient vector at that point indicates the direction where the intensity variation is larger. Besides, the high-level curves of the image function will be in the perpendicular direction of the gradient vector. The edge line that we are looking for does not really coincide with the high level curve of the image function, except for ideal images where the intensity level is constant in the both sides of the edge, as seen in Figure 4. 


\subsubsection{A Computational Approach to Edge Detection}

Computational approach to edge detection [12] describes an edge detection and line finding technique. The line finding consists of determining edge magnitude and direction by convolution of an image with a number of edge masks, of thinning and thresholding these edge magnitudes, of linking the edge elements based on proximity and orientation, and finally of approximating the linked elements by piecewise linear segments. The proposed approach was used for object description and road detection [6].

The key steps in the edge detection algorithm are:

- Convolve the given image with masks corresponding to ideal step edges in a selected number of direction.

- Store the magnitude of the convolved output and the direction of the mask giving the highest output at each pixel.

- Retain only those edges whose magnitude is a local maximum (Thinning).

An edge element is said to be present at a pixel if :

- The output edge magnitude at the pixel is larger than the edge magnitude of its two neighbor in a direction normal to the direction of edge.

- The edge direction of the two neighboring pixels are within one unit (30 degree) of that of the central pixel.

- The edge magnitude of the central pixel exceeds a fixed threshold. If the above two conditions are satisfied, the two neighboring pixels are disqualified from being candidate for edges. 
The isolated edge points are linked together to compute boundary segments. The key steps involved here are:

1.Computing the neighbors that each edge is to be linked to. 2.Tracing boundaries using this linking information. 3.Finally approximating the resulting boundaries by piece wise linear segments. In case of two dimensions it has been shown that marking edge points at maxima of gradient magnitude in the gradient direction is equivalent to finding zero-crossings of a certain nonlinear differential operator. The key steps involved in the detection of edge are:

1.Convolve the image with a symmetric Gaussian. 2.Compute directional second derivative zeros to locate edges and use the magnitude to estimate the edge strength.

It has been shown in [12] that when edge contours are locally straight, highly directional operators will give better results than operators with a circular support.

\subsubsection{Laplacian Edge Detection}

Laplacian Edge Detector [8] is a morphing algorithm for edge detection. It

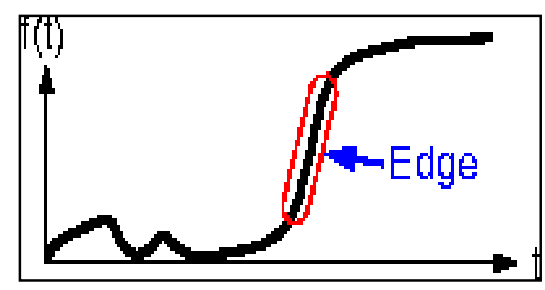

Figure 5: Laplacian Operator 
involves the following steps:

Step 1:

Blur the image: Since there is a need to select edges to perform a morph, there is no need for "every" edge in the image, only the main features. Thus, the image is blurred prior to edge detection. This blurring is accomplished by convolving the image with a gaussian (A gaussian is used because it is "smooth"; a general low pass filter has ripples, and ripples show up as edges)

Step2:

Perform the laplacian on this blurred image [8].

Why is the laplacian used? Let's look at an example in one dimension. Suppose we have the following signal, with an edge as highlighted below.

If we take the gradient of this signal (which, in one dimension, is just the first derivative with respect to $t$ ) we get the situation described in Figure 6 .

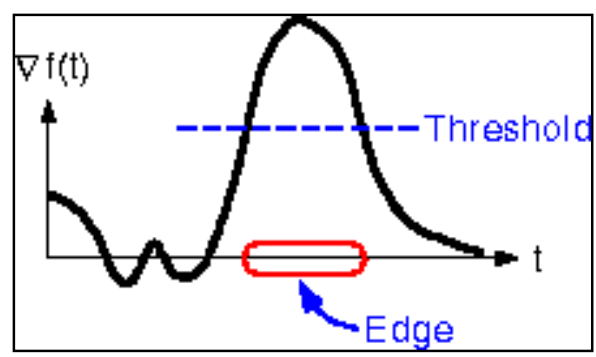

Figure 6: Gradient operators applied to the signal.

Clearly, the gradient has a large peak centered around the edge. By comparing the gradient to a threshold, we can detect an edge whenever the threshold is exceeded (as shown above). In this case, we have found the edge, but the edge has become "thick" due to the thresholding. However, since we 
know the edge occurs at the peak, we can localize it by computing the laplacian (in one dimension, the second derivative with respect to t) and finding the zero crossings.

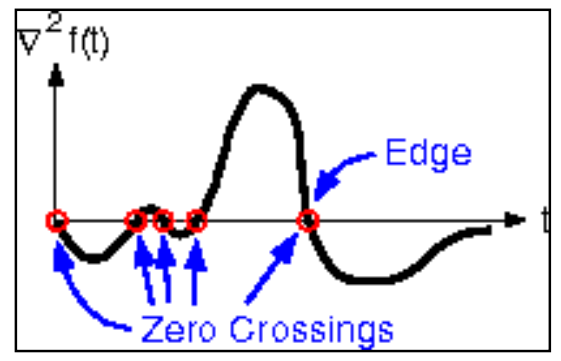

Figure 7: Zero Crossing

Figure 7 shows the laplacian of our one-dimensional signal [8]. The, edge corresponds to a zero crossing, but we also see other zero crossings which correspond to small ripples in the original signal.

To remove these false edges that are produced due to the ripples, we add a step to our algorithm. When we find a zero crossing of the laplacian, we must also compute an estimate of the local variance of the test image, since a true edge corresponds to a significant change in intensity of the original image. If this variance is low, then our zero crossing must have been caused by ripple.

Step 3:

Find the zero crossings of the laplacian [8] and compare the local variance at this point to a threshold. If the threshold is exceeded, declare an edge. 
Step 4:

By applying a median filter [8] the spot noise is removed while preserving the edges. This yields a very clean representation of the major edges of the original image.

\subsection{Image Formats}

Images are stored in various file formats. Image formats being dealt with in this project are:

- DICOM (Digital Imaging and Communications in Medicine).

- TIFF (Tagged Image format).

- Raw (Binary format).

\subsubsection{DICOM}

DICOM(Digital Imaging and Communications in Medicine) standard was created by the National Electrical Manufacturers Association (NEMA) to aid the distribution and viewing of medical images, such as CT scans, MRIs, and ultrasound. A single DICOM file contains both a header (which stores information about the patient's name, the type of scan, image dimensions, etc), as well as all of the image data (which can contain information in 3Ds). This is different from the popular Analyze format, which stores the image data in one file ( $\left.{ }^{*} . \mathrm{img}\right)$ and the header data in another file ( ${ }^{*}$.hdr). DICOM image data can be compressed (encapsulated) to reduce the image size. Files can be compressed using lossy or lossless variants of the JPEG format, as well as a lossless Run-Length Encoding format (which is identical to the packed-bits compression found in some Tiff 
format images). DICOM is the most common standard for receiving scans from a hospital. Figure 9 shows a typical DICOM file with the DICOM image and the DICOM header.

\begin{tabular}{|c|}
\hline 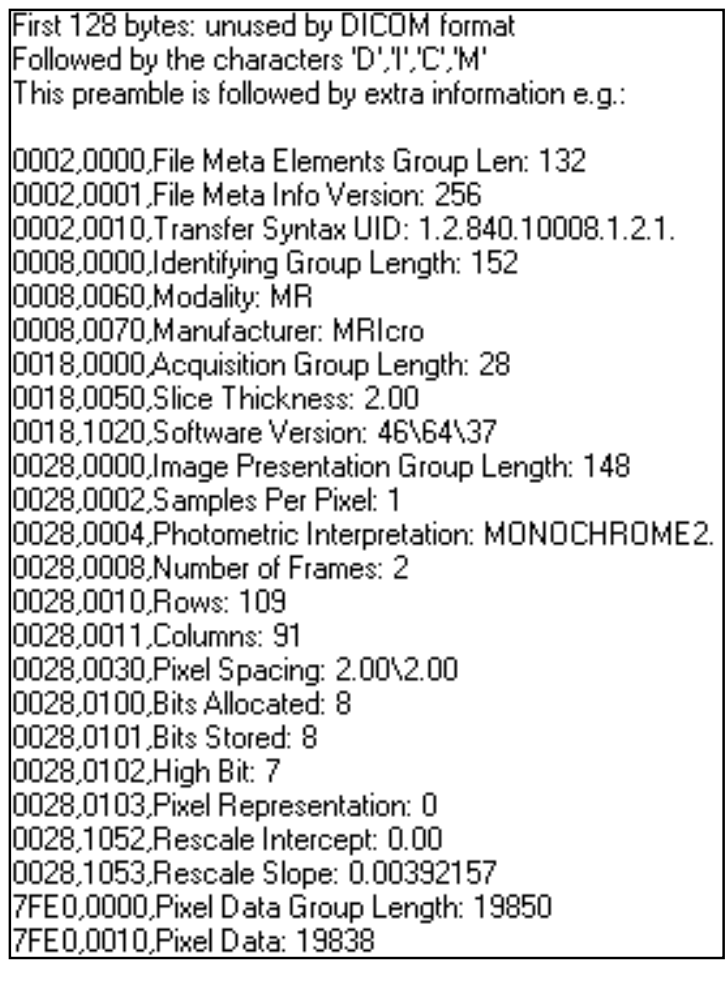 \\
\hline
\end{tabular}

Figure 8: DICOM Header

Figure 8 shows a hypothetical DICOM Header file. In this example, the first 794 bytes are used for a DICOM format, which describes the image dimensions and retains other text information about the scan. The size of this header varies depending on how much header information is stored. Here, the header defines an image which has the 7 dimensions 109x91x2 voxels (three dimensional pixels), with a data resolution of 1 byte per voxel (so the total image 
size will be 19838). The image data follows the header information (the header and the image data are stored in the same file).

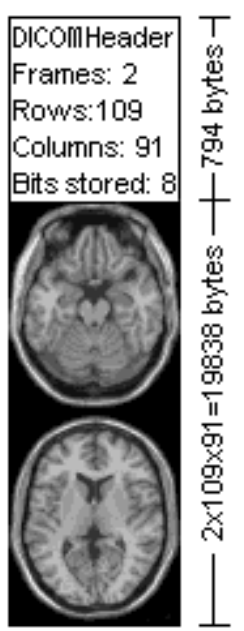

Figure 9: DICOM File

\subsubsection{TIFF}

TIFF is an acronym for Tagged Image File Format. It is one of the most popular and flexible of the current public domain raster file formats [15].

It's main strengths are a highly flexible and platform-independent format which is supported by numerous image processing applications. Since it was designed by developers of printers, scanners and monitors, it has a very rich space of information elements for colorimetry calibration, gamut tables, etc. Such information is also very useful for remote sensing and multispectral applications. Another feature of Tiff which is also useful is the ability to decompose an image by tiles rather than scanlines. This permits much more efficient access to very 
large imagery which has been compressed (since one does not have to decompress an entire scanline).

Theoretically, Tiff can support imagery with multiple bands (up to $64 \mathrm{~K}$ bands), arbitrary number of bits per pixel, data cubes, and multiple images per file, including thumbnail subsampled images.

Features of Tiff format [15] are the following:

- Tiff is capable of describing bi-level, grayscale, palette-color, and full-color image data in several color spaces.

- Tiff includes a number of compression schemes that allow developers to choose the best space or time tradeoff for their applications.

- Tiff is not tied to specific scanners, printers, or computer display hardware.

- Tiff is portable. It does not favor particular operating systems, file systems, compilers, or processors.

- Tiff is designed to be extensible — to evolve gracefully as new needs arise.

- Tiff allows the inclusion of an unlimited amount of private or special-purpose information.

\subsubsection{Raw (Binary Format)}

The Raw Pixel [25] format is useful for applications that need direct access to the pixel data without the burden of the complex computations required to determine the location of pixels within a compressed data stream. This simplifies reading the image for applications that are performing pixel-oriented image processing, such as filtering and edge detection. This format is even more useful to applications that need to write data back to the image. This is because 
changing even a single pixel in a compressed image can have implications for the entire image stream, providing an uncompressed format enables applications to write pixel data directly, and later compress the image with a single process command.

A Raw Pixel image consists of a 4-byte image identifier, followed by a 30byte image header [25], followed by an arbitrary gap of zero or more bytes, followed by pixel data. Raw Pixel images are never color-mapped, and therefore do not contain color lookup tables. 


\section{Chapter 3: 3D Reconstruction from CT}

This chapter describes the reconstruction methods adopted to generate 3D model of the human skeletal leg. Edge detection techniques used in this are similar in concept to the laplacian edge detector described in Chapter 2.

\subsection{Our Approach}

We start by obtaining the Raw(binary) image of the CT scan. The CT originally is in the Dicom format, which needs conversion to Tiff format and then to Raw format. Then we extract the $X, Y$ and $Z$ coordinates of the bone-boundary from the Raw image. We then plot these coordinates to obtain the 3D model. The various steps involved are as follows:

a. Conversion of CT's from Dicom to Tiff format.

b. Processing the images in Tiff format to obtain the Raw or binary format. Using this format we perform edge detection and identify the bone boundary.

c. Extraction of $X$ and $Y$ coordinates from the binary images.

d. Extraction of $Z$ coordinates from the header of the Dicom files.

e. Building the 3D model.

f. Cutting slices on the 3D model.

\subsection{Input Data}

Before we define the process of reconstruction, we present the input formats that were used. CT data of the human skeletal leg is used to perform the reconstruction. CT measures the attenuation of the $x$-rays as they pass through 
the body. CT's comprises of the actual images (unlike images generated from $\mathrm{x}$ rays) of the cut cross-sections of the scanned region. Figure 10 shows a sample of the CT Scan images that were used as input for the 3D modeling. A total of 9 specimen knees were modeled in 3D during the course of this research project. Each of the specimens contained 350 to 470 CT images.

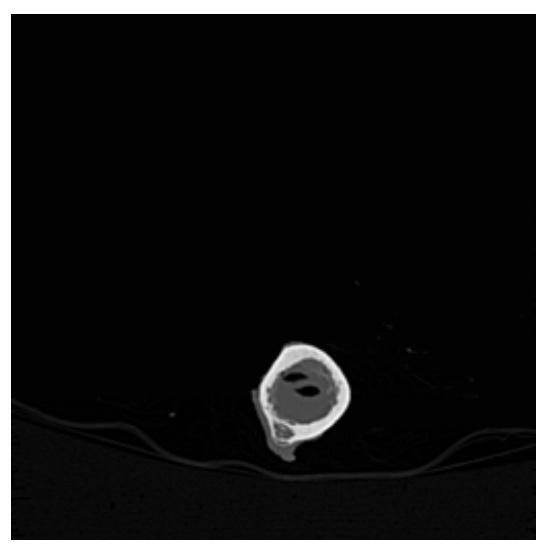

(a)

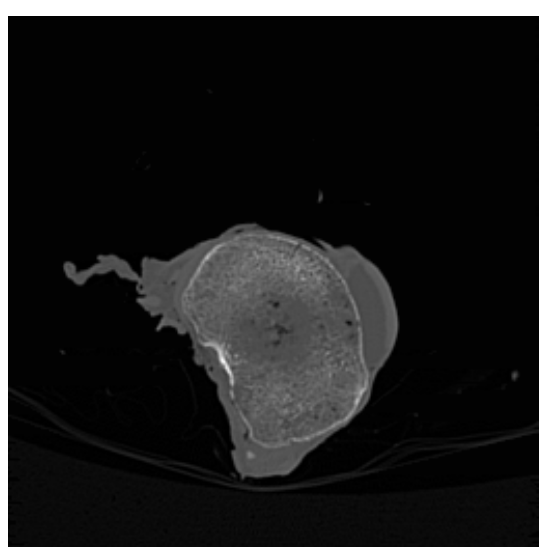

(b)

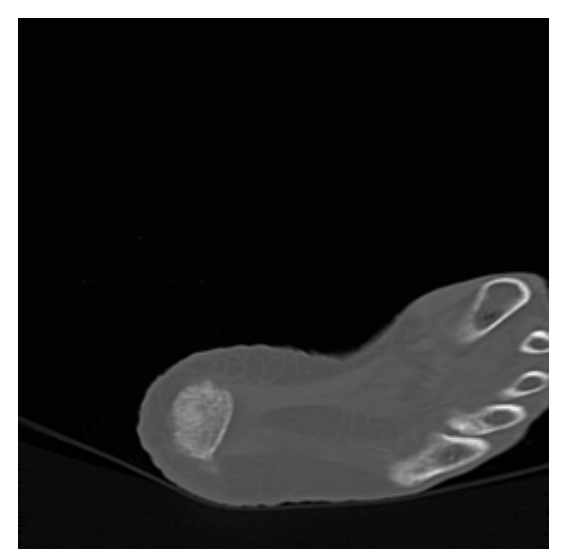

(c)

Figure 10: Sample of CT scans.(a) femoral region (b) knee region (c) foot region 


\section{The 3 Dimensional Reconstruction Technique}

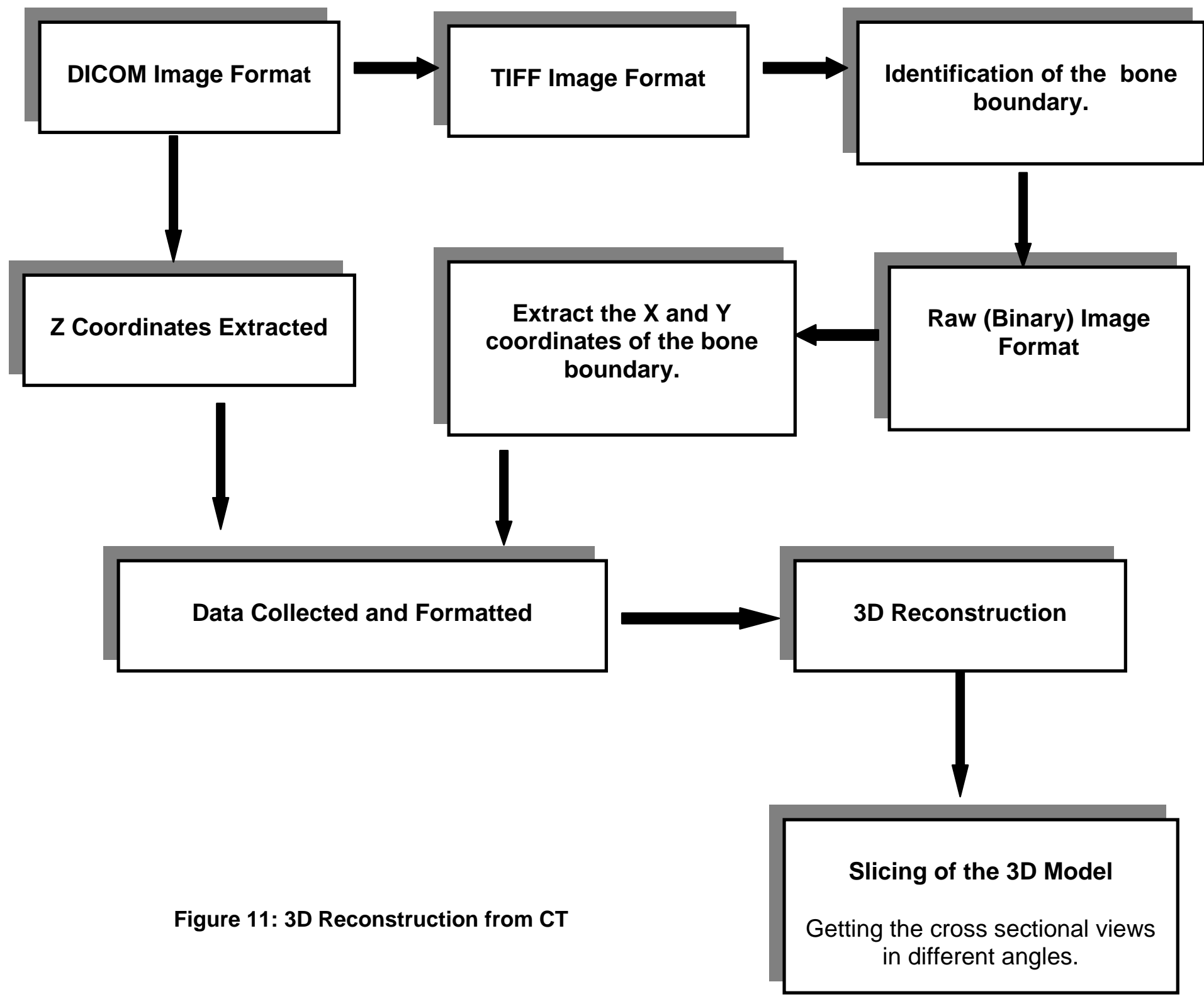




\subsection{Three dimensional reconstruction from CT scans}

The entire reconstruction technique is summarized in Figure 11. Here are the details of the seven steps involved in the 3D reconstruction of the human skeletal leg from CT scans.

\subsubsection{Conversion of CT's from Dicom to Tiff:}

The main strengths of Tiff over Dicom are listed below:

- Tiff is highly flexible and platform independent.

- Tiff is capable of describing bi-level, grayscale, palette-color, and full-color image data in several color spaces.

- Tiff includes a number of compression schemes that allow developers to choose the best space or time tradeoff for their applications.

- Tiff has the ability to decompose an image by tiles rather than scanlines. This permits much more efficient access to very large imagery which has been compressed (since one does not have to decompress an entire scanline).

For the above reasons and many more (see chapter 2 for more details), we choose Tiff over Dicom.

The conversion from Tiff to Dicom conversion is done using Photoshop(an image editing software) and MacroExpress. Photoshop has a Dicom plugin installed in order to read in the Dicom files provided to us by the Radiology Department. The MacroExpress is an application that allows macros to be written to perform Windows related operations. Macro using MacroExpress has been 
written to do batch processing of all the images for conversion from Dicom to Tiff format.

\subsubsection{Processing the Tiff Images to obtain the Raw (binary) Images}

This step deals with the detection of the bone boundary. The tool used for this purpose is Scion Image. Static thresholding (where the threshold value is constant and preset) is used to do the edge detection. Macros have been written in the programming language supported by Scion Image. The threshold value (ranging between 1-255) is set interactively. The result of executing the macro is a set of binary images. Figure 12 shows a comparison of a Tiff and Raw Images. The Raw image are obtained by the application of thresholding on the Tiff image.
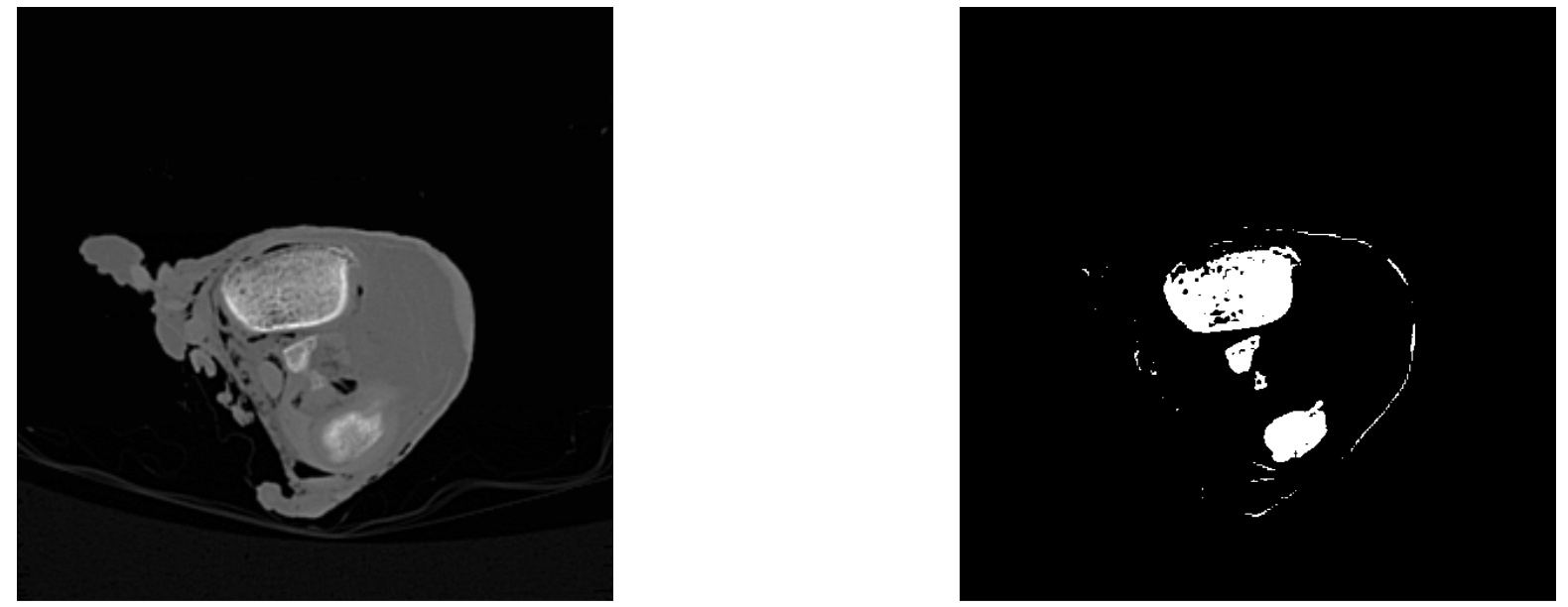

Figure 12: Tiff and Binary Images of the knee region

These Raw images are now ready to be run in the Visual Fortran environment in order to extract the $\mathrm{X}$ and $\mathrm{Y}$ coordinates of the bone boundary. 


\subsubsection{Extraction of bone-boundary coordinates from Binary Images}

We developed a Fortran program which scans every slice and picks up the coordinates of all the points on the boundary. In such a way we separate the bone boundary from the rest of the image.

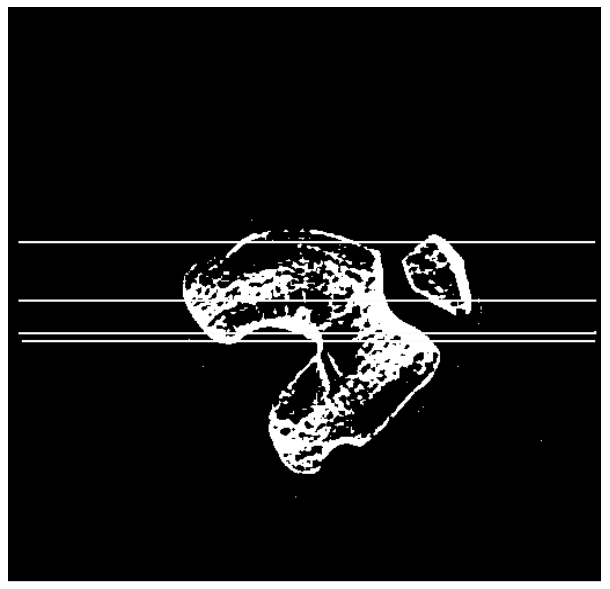

Figure 13: Extraction of the bone-boundary coordinates.

Here are the steps of the algorithm for detecting the $X$ and $Y$ coordinates of the bone-boundary:

Scan the image from left to right and top to bottom.

- Mark all the points where the pixel intensity changes from either a black to white or from white to black. Remember that the Raw image is a bi-level image format, where every pixel is either white or black.

- If consecutive switches (a 'switch' is a change of color from black to a white or vice versa) are very close, say 10 pixel-length, then we neglect the switch, i.e., we do not register the point which was responsible for that switch. This is done in order to reduce the number of unnecessary switches.

- All the points registered as $X, Y$ coordinates are written into a text file. 
- This text file with all the $X, Y$ coordinates along with the $Z$ coordinates, obtained from the CT Header file, forms the input to the 3D reconstruction program.

Figure 13 shows a sample of the edge extraction program output, where the horizontal lines are the scan lines which trace the screen from top to bottom. Whenever there is a switch between a white and black pixel, the coordinates of that pixel are marked. In this process a few of the unwanted switches (like the ones in the bone interiors) are marked, but they can be cleaned up later using another program.

\subsubsection{Extraction of $Z$ Coordinates}

The $Z$ coordinate information is located in a fixed location in the header file of the Dicom format. In the header file shown in Figure $14,+0658$ is the $Z$ coordinate value. 


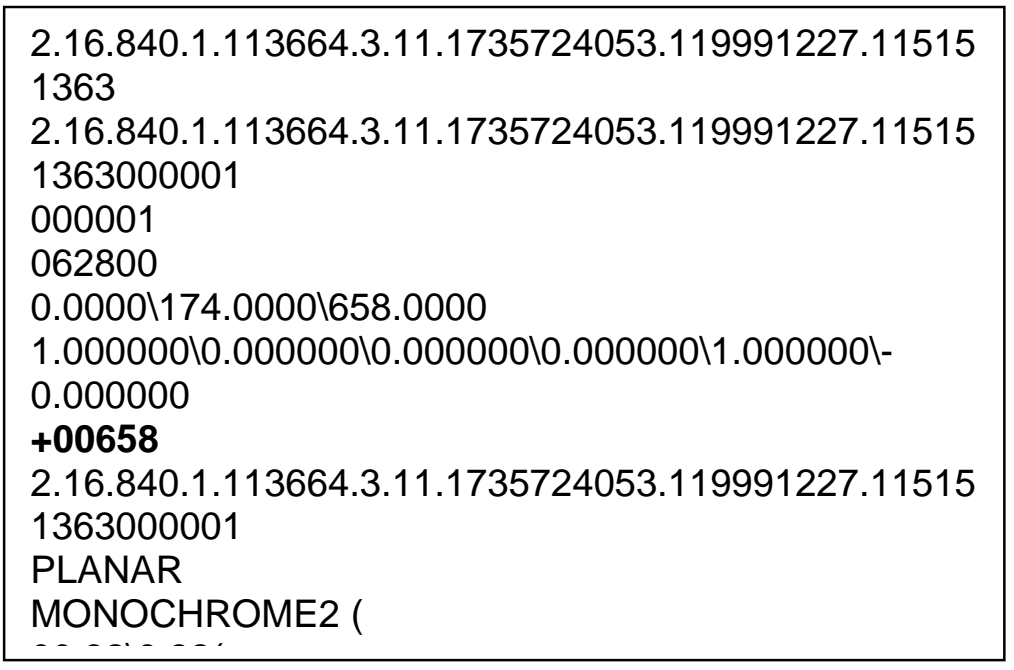

Figure 14: A part of the Dicom Header with the $Z$ value

The DICOM header file is opened in a text format and the $Z$ coordinate value is read into a text file.

\subsubsection{Building the 3D Model}

The $X, Y, Z$ coordinates are plotted in 3D space. A provision is given to the user to plot all points or to just plot in decrements of a factor of ten, i.e., a first decrement would plot only one-tenth the total number of points while the second decrement would plot only one-hundredth the total number of points. The idea is to give the user the option to reduce the number of points so that the time taken to do operations like rotation, magnification, translation etc. would be a lot less than when dealing with the full number of points. Figure 16 shows a sample of the reconstructed model.

Formatting the Data: The data is stored in 'unformatted-random file' form. Storing the data in the 'unformatted' style saves a lot of processing time that is involved when we use 'formatted' style. The access to the elements of a random 
file is faster than that of the sequential storage. Therefore we use 'unformattedrandom' files to store the data.

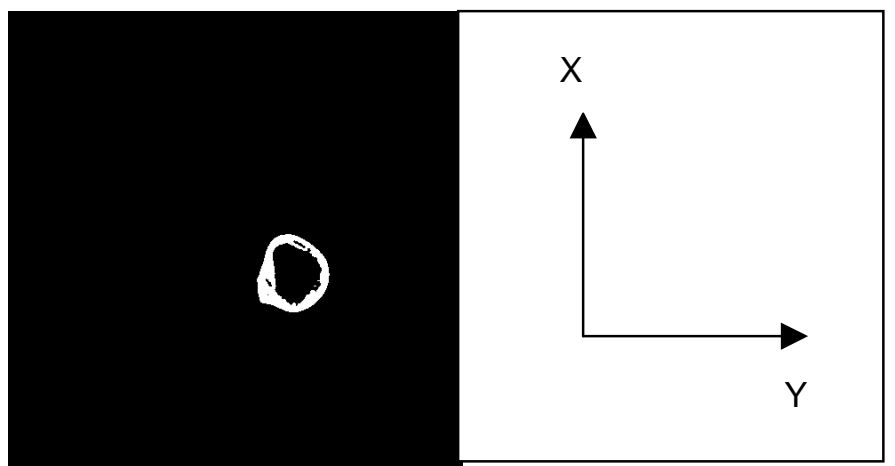

Figure 15: Relation between the cross sectional image and the $X, Y$ coordinate system

The Data is then read from the file into a set of three arrays, one for $X, Y$ and $Z$ each. Each of the coordinates are then displayed in the $Y$ and $Z$ plane to

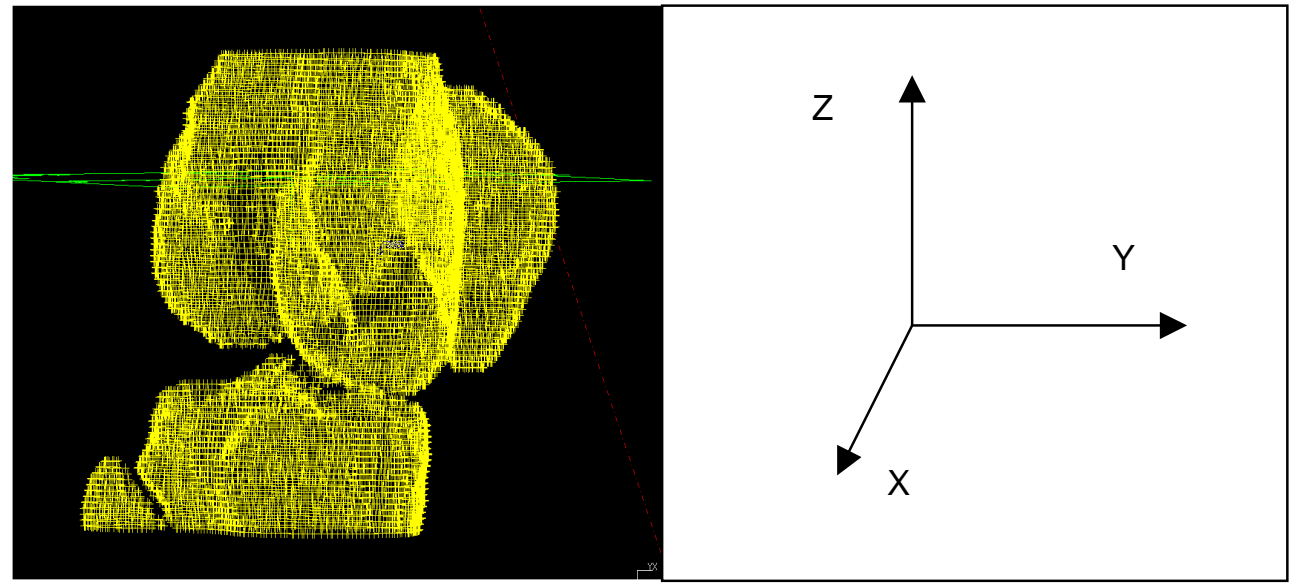

Figure 16: Figure shows the 3D model of the human knee W.R. to the $X, Y$, $Z$ coordinate system.

obtain the 3D model on a 2D monitor screen. Figures 15,16 give the directions of the three axes of the coordinate system relative to the $3 \mathrm{D}$ model as well as the CT Scan. The different operations that are possible with the 3D model are discussed in the next chapter. 


\subsubsection{Cutting Slices on the 3D Model}

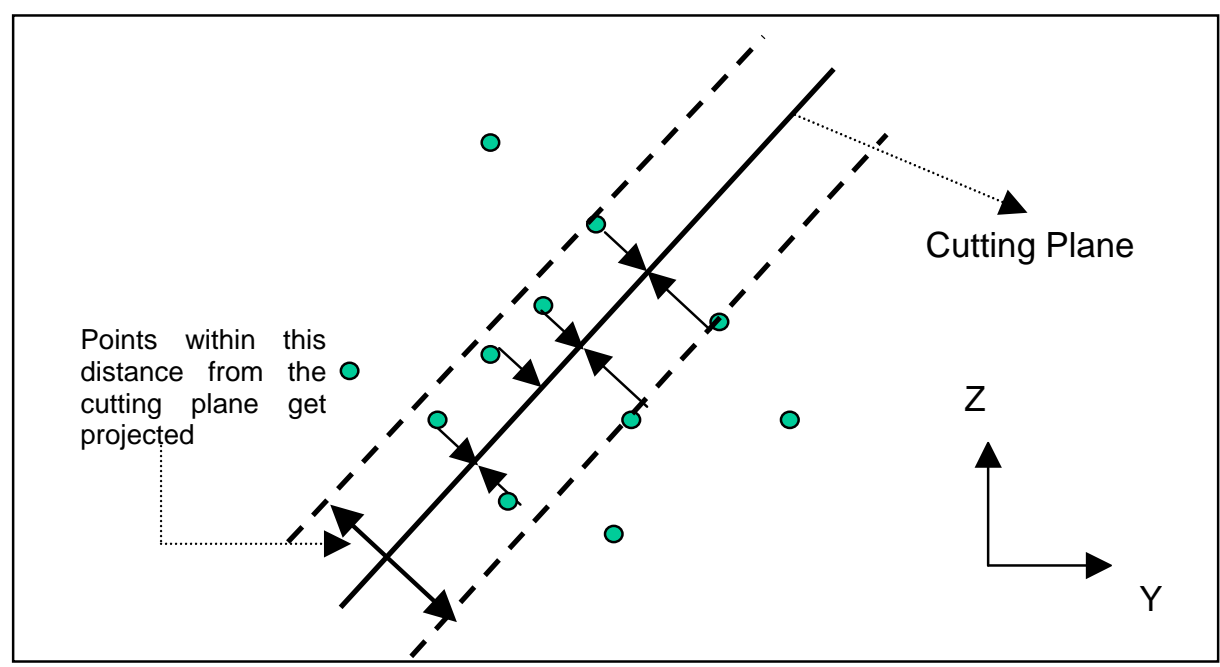

Figure 17: The Slicing technique illustrated

This technique of cutting slices through the 3D model gives us a chance to look at the cross-sectional view of the bone structure. A fair amount of the inside details are also revealed in the process.

The cutting plane is defined by the direction cosines of the normal vector. These direction cosines are provided by the user. To get a cross sectional view we project the coordinate points that are within one millimeter distance on either side of the plane, onto the cutting plane(see Figure 17). Now what we obtain is the $2 \mathrm{D}$ view of the cross section. The points that are viewed are in the $\mathrm{XZ}$ plane. There is also a provision to increase the number of points that can be projected onto the cutting plane by increasing the allowable distance on either side of the plane.

Our 3D model is a collection of points and therefore lacks a surface. We project points onto the cutting plane in order to obtain the cross sectional view 
since the model lacks a surface. There might be a case when the cutting plane may not cut any (or maybe very few) of the coordinate points. By projection we make sure that the above does not happen.

At the time of display the cutting plane is always displayed in the vertical

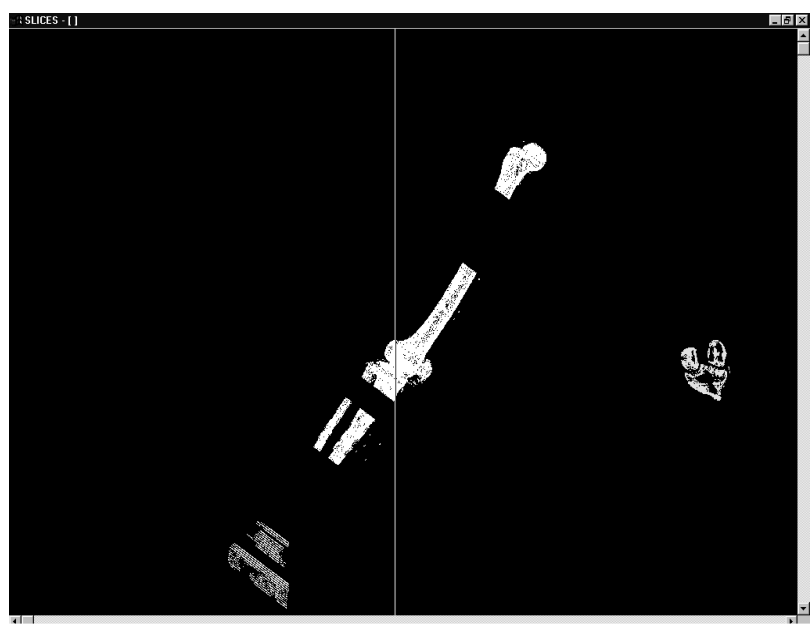

Figure 18: Cross sectional view at the knee region

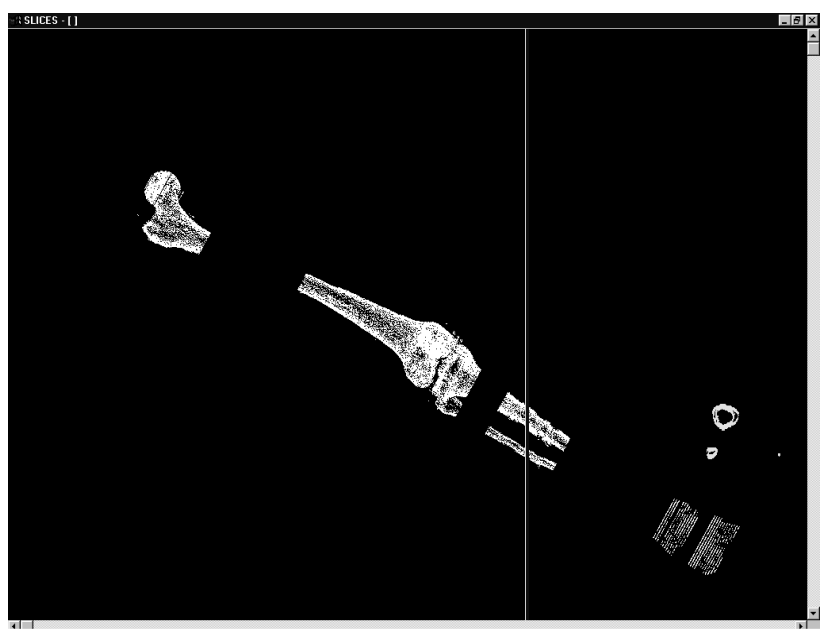

Figure 19: Cross sectional view through the tibia

position i.e. parallel to the $Y$ axis. Keeping the cutting or the slicing plane parallel to one of the axes(here $Y$ axis) simplifies the mathematics involved. The sliced plane that is obtained in displayed in the $X, Z$ plane. Since the sliced plane is perpendicular to the $\mathrm{Y}$ axis, the $\mathrm{X}$ and $\mathrm{Z}$ values when plotted would represent the plane. Therefore we avoid the tedious task of finding projections by keeping the slicing pane perpendicular to the $\mathrm{Y}$ axis.

The mathematics involved in the projection of a $2 \mathrm{D}$ cross sectional plane are as follows:

$$
[\mathrm{X}, \mathrm{Y}, \mathrm{Z}] \longrightarrow[\mathrm{X}, \mathrm{C}, \mathrm{Z}],
$$

where $\mathrm{C}$ is a constant. The slicing plane is given by the equation $\mathrm{Y}=\mathrm{C}$. 
Figures 18,19 and 20 show results of the slicing tool. Figure 18 gives a cross sectional view at the knee region, showing the condyles of the femur

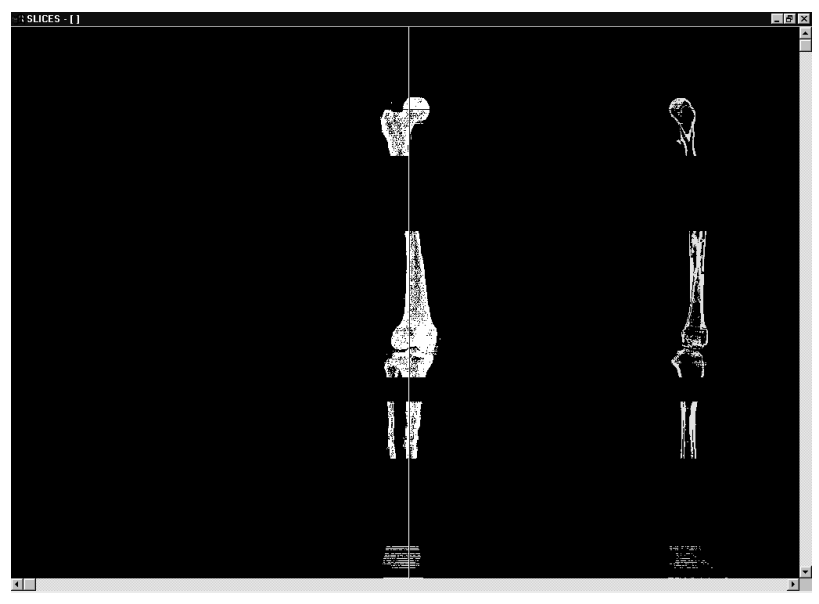

Figure 20: A longitudinal cross section

appearing as small circles. Figure 19 gives a picture of the tibia and fibula. Figure 20 is a longitudinal section of the entire human leg. In all these images (Figures 18, 19 and 20) we display the 2D projection on the right side of the 3D model. The slicing plane is always kept upright. The default display colors for the images are, white for the 3D model, yellow for the $2 \mathrm{D}$ cross sections and blue for the cutting plane. However, these colors can be changed to personal liking. To increase the clarity of the $2 \mathrm{D}$ cross section we need to project more points onto the slicing plane. This is done by increasing the thickness of the slicing plane, which would result in more points being projected onto the cutting plane and thereby more details being visible in the cross sectional view. 


\subsection{The Clean-up Tool:}

The Clean-up tool is a program written in Visual Fortran which helps the user in erasing the unwanted points present in the 3D model. Since the efficiency of the edge detection program is not $100 \%$ we tend to pick up unwanted points as edges. These unwanted 'extra' points show up in the 3D model as 'noise'.

A box is required to be drawn around the set of points which need to be deleted. All the points which are inside the box (as seen in Figure 21) are deleted from the array of coordinates. Figure 21 shows a point $\left(y_{3}, z_{3}\right)$ being deleted by

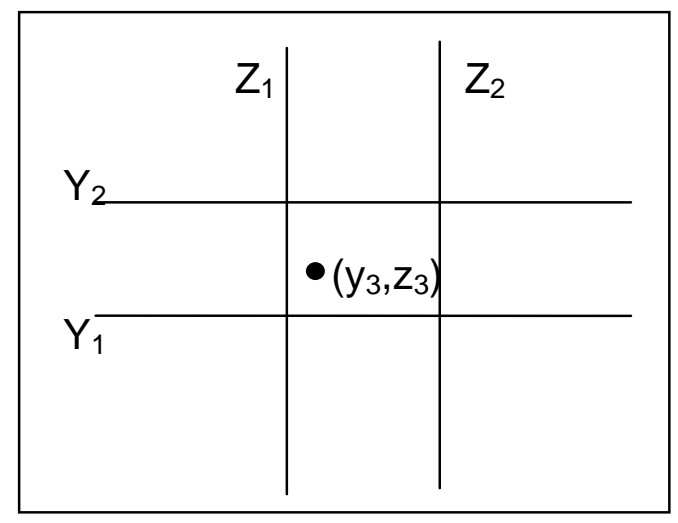

Figure 21: The box which eliminates the all the points inside it.

drawing a box around it. The clean-up box is drawn by independently moving each of the four sides of the box, represented by $Y 1, Y 2, Z 1$ and $Z_{2}$ in Figure 21. Figure 22 shows the Clean-up tool as it appears in the program. It shows the clean-up box drawn around the unwanted points at the vicinity of the 3D model. Condition for a point to be inside the box is:

$$
\mathrm{Y}_{1}<\mathrm{y}_{3}<\mathrm{Y}_{2} \text { and } \mathrm{Z}_{1}<\mathrm{Z}_{3}<\mathrm{Z}_{2}
$$




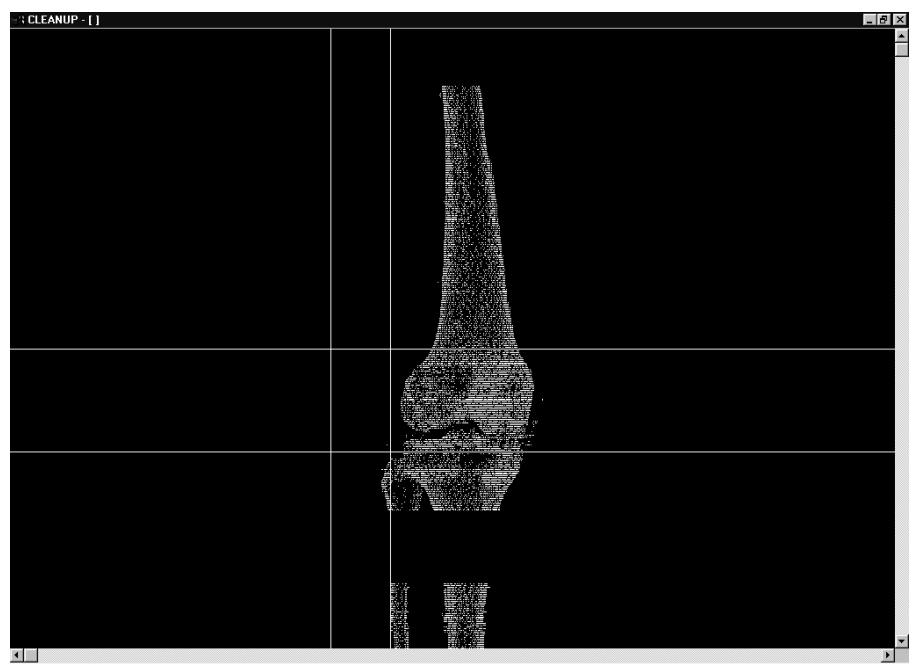

Figure 22: The clean-up box

\subsection{Operations allowed with the reconstruction tool}

The phrase 'reconstruction tool' refers to the program written in Visual Fortran. The reconstruction tool can be used after the bone boundary detection has been done and the $X, Y, Z$ coordinates are plotted. We allow numerous operations on the 3D model that is generated. These operations include:

- Reduction in the number of points displayed.

- Rotation around $X, Y, Z$ directions.

- Translation all directions (up, down, left, right).

- Magnification. 
The implementation of each of these operations is explained in detail in the following subsections.

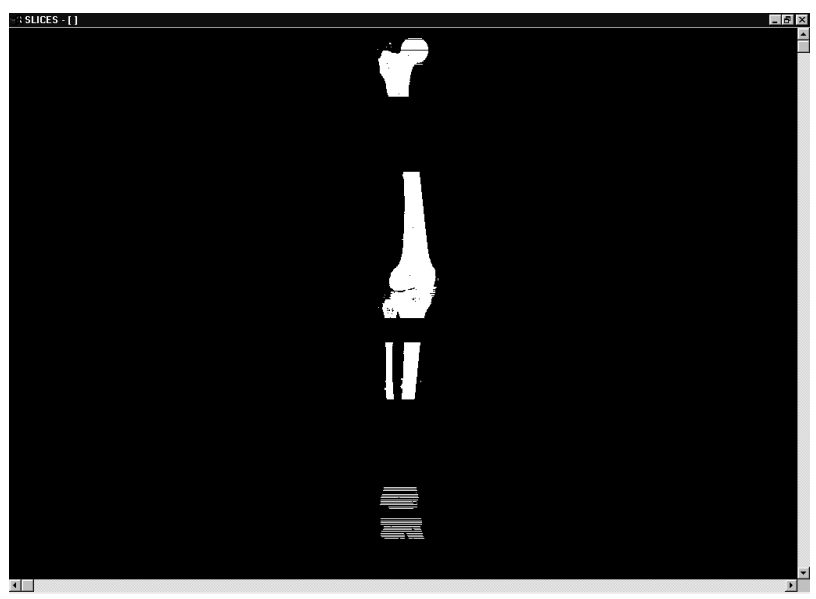

Figure 23: The model with all data points displayed

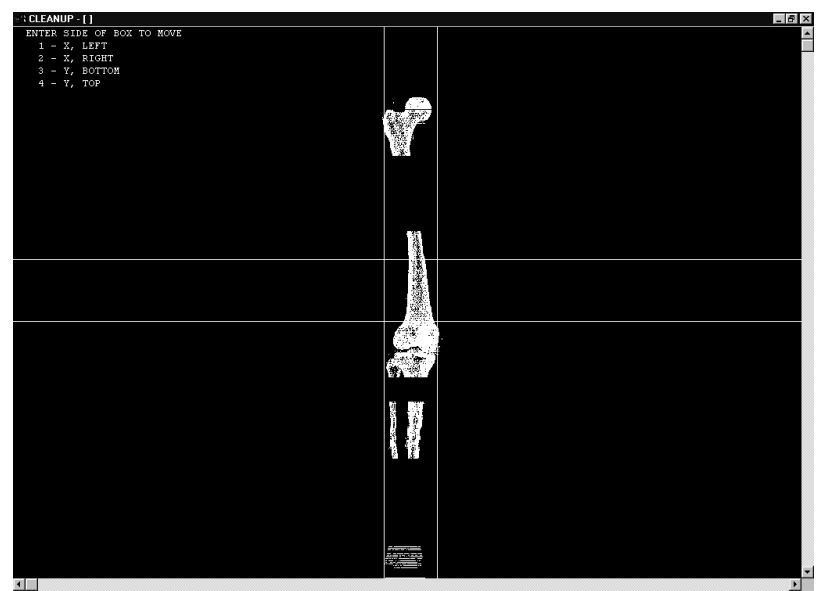

Figure 24: The model with one-tenth of the total number of data points

\subsubsection{Reduced display}

With the reduced number of points operations such as rotation and translation are much faster. For fast results the user is required to reduce the number of points displayed, perform the necessary operations and then revert back to the original number of points to get a better view of the 3D model.

The number of points displayed are reduced by $1 / 10^{\text {th }}, 1 / 100^{\text {th }}$, so on. If the $1 / 10^{\text {th }}$ option is chosen, then we plot every $10^{\text {th }}$ element in the array of coordinates. Figure 23 shows the 3D model displaying all the data points that are available. Implementing operations such as rotation, translation etc., would be very slow with this model owing to the huge number of data points. Figure 24 shows the display of the 3D model with reduced number of points(reduced to 
$\left.1 / 10^{\text {th }}\right)$. This model in Figure 24 would be a faster than the one in Figure 23 with operation such as rotation and translation.

\subsubsection{Rotation}

Rotation is allowed around $X, Y, Z$ axes. The rotation of the 3D model in the desired directions allows the user to get a better view of the 3D model. The rotation is implemented by the multiplication of the coordinate arrays with the rotation matrix as in,

$$
\left[\begin{array}{l}
X \\
Y \\
Z
\end{array}\right]=\left[\begin{array}{l}
x \\
y \\
z
\end{array}\right]{ }^{*} \mathrm{Rx},
$$

where $[X, Y, Z]$ are the resultant coordinates of $[x, y, z]$ after rotation around the $X$ axis.

The rotation matrices are defined as,

$$
\begin{aligned}
& \mathrm{Rx}=\left[\begin{array}{ccc}
1 & 0 & 0 \\
0 & \cos \theta x & \sin \theta x \\
0 & -\sin \theta x & \cos \theta x
\end{array}\right] \\
& \mathrm{Ry}=\left[\begin{array}{ccc}
\cos \theta y & 0 & \sin \theta y \\
0 & 1 & 0 \\
-\sin \theta y & 0 & \cos \theta y
\end{array}\right] \\
& \mathrm{Rz}=\left[\begin{array}{ccc}
\cos \theta z & \sin \theta z & 0 \\
-\sin \theta z & \cos \theta z & 0 \\
0 & 0 & 1
\end{array}\right]
\end{aligned}
$$




\subsubsection{Translation}

Translation allows the user to move the $3 \mathrm{D}$ model in all the four directions(up, down, right, left). The translation matrix is generated using 'homogenous coordinates'.

The $3 \times 3$ matrix is converted to a $4 \mathrm{X} 4$ matrix in order to incorporate both

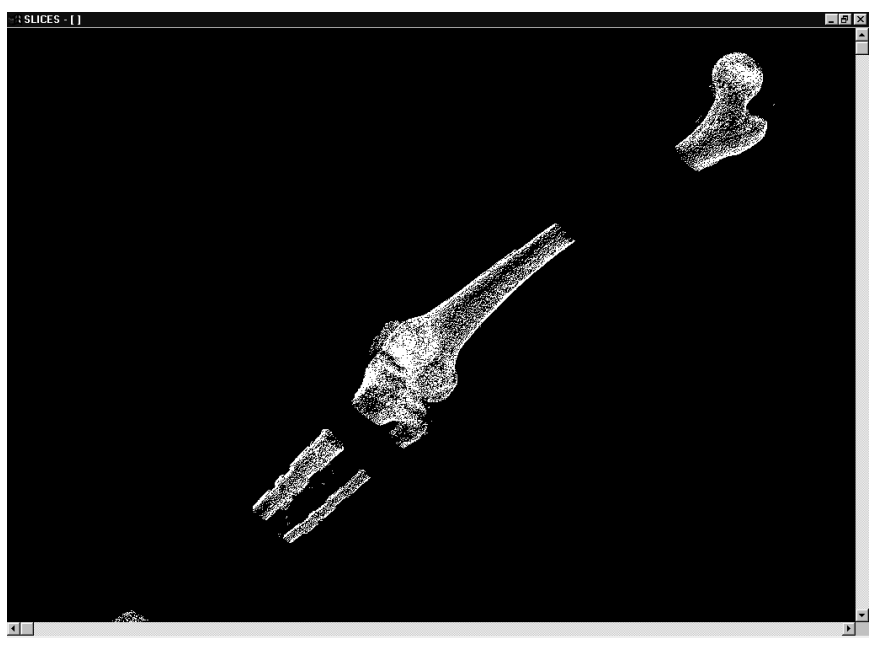

Figure 25: A result of both rotation and translation

translation and rotation in the same transformation matrix. The resultant $4 \mathrm{X} 4$ matrix is known as the 'Homogenous Coordinates', shown here:

$$
\mathrm{Tx}=\left[\begin{array}{cccc}
1 & 0 & 0 & \Delta x \\
0 & \cos \theta x & \sin \theta x & \Delta y \\
0 & -\sin \theta x & \cos \theta x & \Delta z \\
0 & 0 & 0 & 1
\end{array}\right],
$$

where $\mathrm{Tx}$ is the transformation matrix for the $\mathrm{x}$ axis.

A linear transformation, i.e., a translation would look like this in linear equation form, $Y_{1}=Y_{2}+\Delta y$, where $Y_{1}$ is the result of translating $Y_{2}$ in the $Y$ axial direction by a factor of $\Delta y$. 


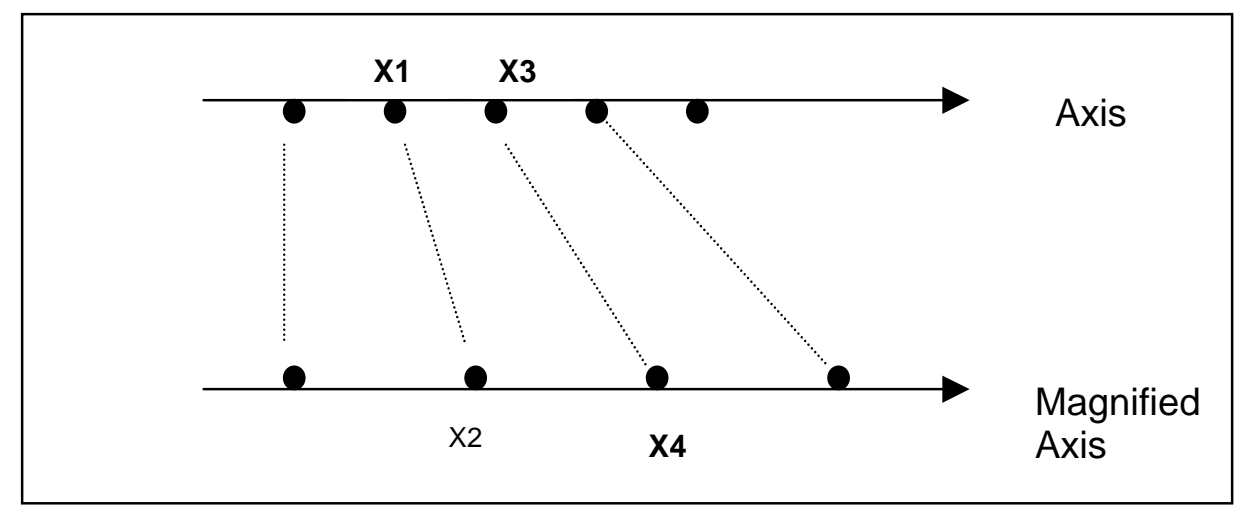

Figure 26: Magnification achieved by altering the scale factor

Figure 25 shows the results of both magnification and rotation on the 3D model. By doing a combination of translation, rotation and magnification we can view the interesting portions of the 3D model as depicted in Figures 27(a) and $27(b)$.

\subsubsection{Magnification}

Magnification allows the user to magnify and see more details of the 3D model. Here is how the 3D magnification is done:

Figure 26 shows the change of scale for plotting the data points when the magnification tool is used. The scale factor on the axes is increased and thereby the coordinate points of the human skeletal leg get plotted at an increased distance, and thereby the picture is magnified. Mathematically, the magnification can be represented as follows(see Figure 26 for references):

$$
X_{1}=m * X_{2} \text { and } X_{3}=m * X_{4} \text {, }
$$

Where $m$ is the magnification factor with the following definition, $m>1$ for magnification and $m<1$ for minimization. When $m=1$ we would obtain the image 
in its original size. $X_{1}$ and $X_{3}$ correspond to points on the original axis, while $X_{2}$ and $\mathrm{X}_{4}$ are points on the magnified axis(see Figure 26). The default value for the magnification factor $(\mathrm{m})$ is 0.05 (or $5 \%$ ) for every click of the magnification button.

Figures 27(a)\&(b) shows a couple of magnified images of the 3D model. It can be seen that the density of the data points in Figures $27(a) \&(b)$ is less due to the magnification of the image. A combination of translation and magnification would result in images $27(a) \&(b)$. Figure $27(a)$ is a zoom-in image of the kneejoint region while Figure 27(b) shows the magnified image of the femoral head.

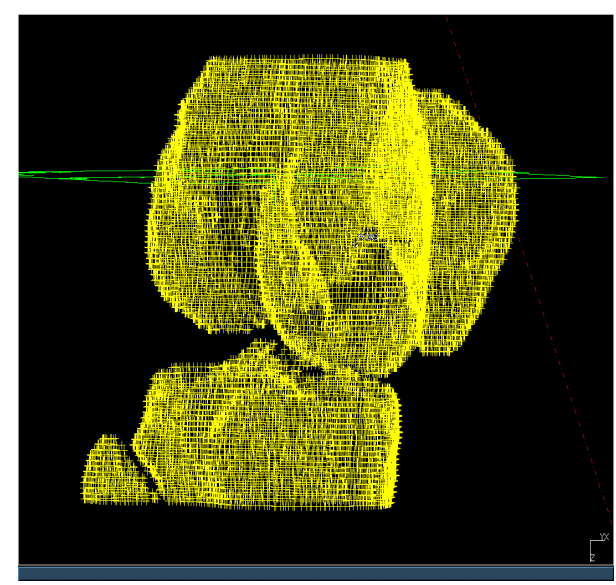

(a)

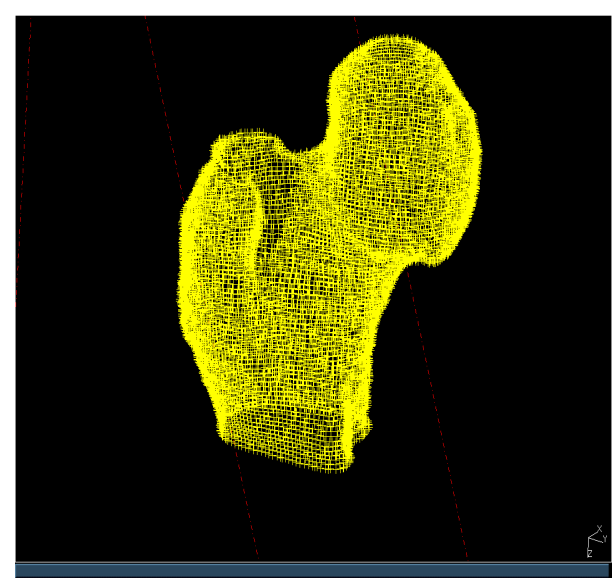

(b)

Figure 27: The results of magnification 


\section{Chapter 4: Conclusion}

This chapter summarizes the work that has been done in the context of this research project, and outlines avenues for future research work. It also throws light on some of the issues and challenges that were dealt with in this project.

\subsection{Summary}

Edge Detection Techniques play a very important role in research in the field of medicine. In the field of orthopedics, edge detection techniques are being used in the reconstruction of the skeletal system from inputs such as MRI and CT Scans. These reconstructed models form a basis for research as well as preoperative procedures.

In this thesis we developed a very simple process for the three dimensional reconstruction of the human knee starting from computed tomography $(\mathrm{CT})$.

The first phase of the research involves the conversion of the images from Dicom format to a more widely used Tiff image format. The next stage is the use of edge detection procedures to extract the edge of the bone. The challenge here lies in the separation of the bone from the surrounding flesh and muscle. The bone boundary detection is the main focus of the thesis. After the bone edge has been determined we then proceed to extract the coordinates of the bone boundary. These coordinates are then plotted in 3 dimensional space to give the 
3D model of the human skeletal leg. A number of operations are allowed on the $3 \mathrm{D}$ model that is obtained.

The main goal of this thesis has been to identify a workable process of reconstructing the human skeletal leg from CT Scans in the shortest possible time. This goal has been achieved to a good extent. There has been a slight compromise in the accuracy of the reconstruction in order to obtain a faster method of reconstruction. But this inaccuracy did not affect the on going research in knee kinematics.

\subsection{Future Work}

Here are a few avenues for future work:

1. The different modules of this project should be combined into a single unit, so that all the different steps can be performed by executing a single tool. Also a GUI (Graphics Users Interface) should be developed for this unit.

2. To obtain a better and a faster method for the conversion of Images from Dicom to Tiff format. Probably write a program in either $\mathrm{C} / \mathrm{C}++$ (or any other language) for the above job. This would, however, involve a thorough understanding of both the Image Formats, their internal structure and their inter-dependencies. Such a program would allow a faster batch job to be run in order to convert all the CT's from Dicom to Tiff.

3. Fine-tune the edge detection technique in order to pick up finer details in the Image so that the tool can be used in the making of physical models of the patients knee. This allows a though understanding and better pre-operative preparation for the doctors. 
4. Make the Thresholding Dynamic. This would involve the setting of a separate threshold value for each and every CT image, depending on the nature of the CT image. This would involve the calculation of the standard deviation from the average pixel value of the image. 


\section{References}

1. Kas, M., Witkin, A., Terzopolous, D., "Snakes: Active Contour Models," International Journal of Cognitive Vision, 1:321-331, 1987.

2. Trujillo, Augustin., "Thin Edge Detection," Proceedings of Intl. Conference on Image Analysis and Processing, Spain, 1991.

3. Alvarez, L., Lions, P. L., and Morel J. M, "Image selective smoothing and edge detection by nonlinear diffusion," SIAM Journal of Numerical Analysis., 29(3):845-866, 1992.

4. Norman, T. L., Vashishth, D., and Burr, D. B., "The Effect of Groove in Bone Fracture Toughness Testing," Journal of Biomechanics, 25(12):1489, Dec., 1992.

5. Woltring, H.J., Huiskes, R., De Lange, A., and Veldpaus, F.E., "Finite centroid and helical axis estimation from noisy landmark measurements in the study of human joint kinematics," Journal of Biomechanics, 18:379-389, 1985.

6. Marr, D., and Hildreth, E., "Theory of Edge Detector," in Proceedings of Royal Society, Sec B. 207:187-217, 1980.

7. He, X., and Hintz, T., "Multi-scale detection based on Spiral Architecture," in 1998 International Computer Symposium, 159-165, Taiwan, 1998.

8. Claypoole, Roger., Lewis, Jim., Bhasyam, Srikrishna., Kelly, Kevin., Image Morphing, Technical Report, Dept. of Electrical Engineering, Rice Univ., http://www.owlnet.rice.edu/ elec539/Projects97/morphjrks/laplacian.html, 1997. 
9. Schroeder, W., Martin, K., Lorensen, B., The Visualization Toolkit, Prentice Hall Publication, 1997.

10. Gonzalez, C.R., Woods, R. E., Digital Image Processing, Addison Wesley Publications, 1992.

11. Sonka, M., Hlavac, V., Boyle, R., "Image Processing, Analysis, and Machine Vision," Technical Report, 1998.

12. Canny, J. F., "A computational approach to edge detection," IEEE Transactions on Pattern Analysis and machine Intelligence, 8(6):679-698, 1986.

13. Visual Numerics Inc., PV-Wave for Windows Development Environment," Technical Report, Http://www.vni.com/products/wave/index.html, 1999.

14.Scion Corporation., User Manual, www.scioncorp.com/tech_support.html, 1999.

15. Adobe Developers Association., "TIFF Revision 6.0", Http://www.adobe.com/Support/TechNotes.html, 1992.

16. Hari Kiran Boddupalli., "Tissue Thickness Measurement Tool for Craniofacial Reconstruction," Master's Thesis, Dept. of Computer Science and Electrical Engineering, West Virginia University, 1999.

17. Laine, H. J., Kontola, K., Lehto, M. U., Pitkanen, M., "Image Processing for femoral endosteal anatomy detection: description and testing of a computed tomography based program," Physics in Medicine and Biology, 42(4):673-89, 1997. 


\section{APPENDIX A}

Here is the list of all the software tools that were made use of in this research work.

- Scion Image: Acquisition and Analysis Software

- Digital Visual Fortran: An environment for programming in Fortran Programming language.

- PhotoShop 5.5: An image editing software from Adobe.

- Macro Express Editor: A Macro script editor.

Here is a brief description of these tools.

\section{Scion Image}

Scion Image [14] for Windows is the Windows version of Scion Image, which is in turn a version of the popular Macintosh program, NIH Image, written at the National Institutes of Health. Scion Image may be used to capture, display, analyze, enhance, measure, annotate, and output images. Scion Image extensively supports all Scion frame grabber boards, and provides a powerful and complete image acquisition environment.

Scion Image for Windows supports color and grayscale image capture with Scion frame grabbers. It includes advanced capturing capabilities such as frame averaging and summation, frame sequence capture, and on-chip integration support. 
Scion Image is available free of charge. Scion provides full technical support to users of Scion Image. Please note that Scion Image for Windows is currently in beta release and cannot be considered bug free. Scion Image for Windows is available in Windows 95, Windows 98, Windows NT and Windows 2000 versions.

\section{Digital Visual Fortran}

DIGITAL Fortran is now Compaq Fortran - the Gold Standard in Fortran Compilers for Windows. Everything is GUI based which makes writing programs, compiling them and running them a lot easier than the command line interface.

\section{Photoshop}

Helps in image editing with powerful features . Delivering the broadest and most productive toolkit, Photoshop helps you explore your creativity, work at peak efficiency, and achieve the highest quality results across all media.

\section{Macro Express Editor}

What is a macro?

A macro is a set of commands that can be played back at will to perform a given task. 
Macro Express is a utility that allows you to create macros manually or by

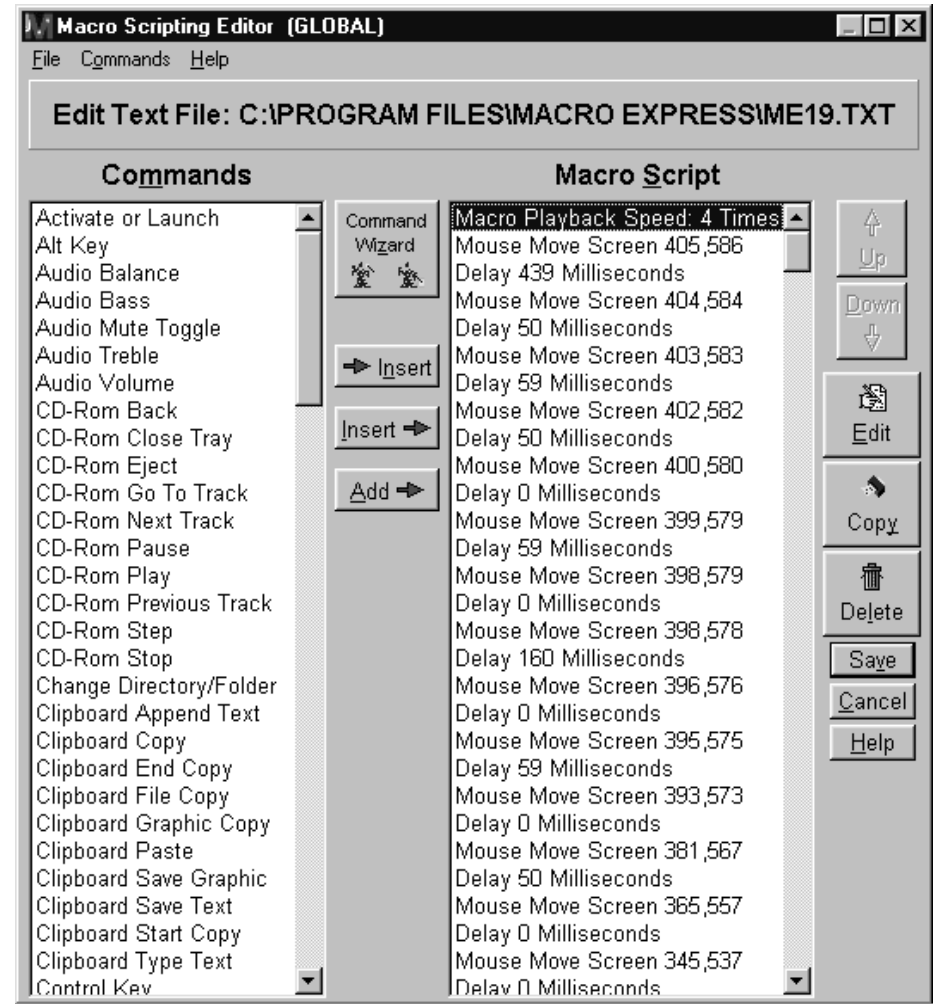

Figure 28: Macro Scripting Editor

recording them. Quick Wizards are available making creation of macros an easy

task. Macros can also be created and edited with a powerful, yet easy "Scripting Editor" (Figure 28) that allows you to see each step and command of your macro. Each individual command can be modified, deleted, copied or moved around in the script. A command wizard is also available to step you through the process of creating your macros. 


\section{APPENDIX B}

\section{Issues and Challenges}

Few of issues with the reconstruction techniques are discussed in the following subsections.

Localization

During the application of thresholding, when a group of high valued pixels (pixel value higher than that of the threshold) are surrounded by a circle of lower valued pixels (whose values are lower than the threshold value) then the edge detection technique could fail as the contours get localized.

The solution to this problem was suggested by Hildreth and Canny in their respective methods, where disconnected contours are not allowed [6].

Unwanted edges

In the process of picking up the required edges, one is bound to pick up 'extra' edges which are not required. The improved edge detection methods use techniques to eliminate these 'extra' edges. One such technique is the linecontour or the connected contour technique. In this technique all the points that join to form a line (or contour) are kept while isolated points are removed. This makes sense since what we are looking for are edges and edges are nothing but connected contours. 


\section{Thresholding}

It is very difficult to pick up a right value for the threshold. A correct threshold would be that value which would give the best results for the edge detection, that which records only the most relevant information from the image(in this case, the edges).

There are many reasons that make it difficult to choose one particular value as the threshold value:

1. The CT themselves differ a lot in their intensity levels. The threshold value which works for one CT may not work for another owing to the difference in their intensity levels. The intensity levels of the CTs vary dependent on the CT Scanner's photon energy settings at the time of the scan.

2. The details that are required to be identified may be different in different applications. In our research, we have different threshold values for identifying the inside information of the bone structure, while another value for the threshold would register the external bone structure. So, it becomes important to decide on the interesting part of the image before setting the threshold value.

The solution to the above problems would be the 'dynamic thresholding'. Dynamic thresholding calculates an appropriate threshold value for the particular image. This calculation is done on the fly using specific properties of the image such as 'average intensity level', 'standard deviation of the intensity levels', etc.

\section{Number of data points}

The reason for the huge size of the data points is due to the 'extra switches' 
that takes place inside the bone boundary. In other words, it is the 'internal switches' of the bone that result in the immensely large number of data points. We reduce the number of points picked up (inside the bone) by the following two methods,

1) Switches that are very close together are ignored. This way we do not miss any of the switches that may occur between an inner point and the outer point (point which lies on the bone boundary).

2) Instead of taking CT's at $1 \mathrm{~mm}$ spacing we take them at $2 \mathrm{~mm}$ or $3 \mathrm{~mm}$ spacing. This way we reduce the number of data points to half or one-third, but at the cost of bone details.

The issue of large data points comes only at the time of display of the 3D model. If fast and quick display of the model is not a criterion then the huge number of data points should not be a concern at all. 